\title{
A globally convergent matricial algorithm for multivariate spectral estimation
}

\author{
Federico Ramponi, Augusto Ferrante and Michele Pavon
}

\begin{abstract}
In this paper, we first describe a matricial Newton-type algorithm designed to solve the multivariable spectrum approximation problem. We then prove its global convergence. Finally, we apply this approximation procedure to multivariate spectral estimation, and test its effectiveness through simulation. Simulation shows that, in the case of short observation records, this method may provide a valid alternative to standard multivariable identification techniques such as MATLAB's PEM and MATLAB's N4SID.
\end{abstract}

\section{Index Terms}

Multivariable spectrum approximation, Hellinger distance, convex optimization, matricial Newton algorithm, global convergence, spectral estimation.

\section{INTRODUCTION}

ARMA identification methods usually lead to nonconvex optimization problems for which global convergence is not guaranteed, cf. e.g. [33], [39], [40], [11]. Although these algorithms are simple and perform effectively, as observed in [40, p.103], [32, Section 1], no theoretically satisfactory approach to ARMA parameter estimation appears to be available. Alternative, convex optimization approaches have been recently proposed by Byrnes, Georgiou, Lindquist and co-workers [3], [30] in the frame of a broad research effort on analytic interpolation with degree contraint, see [1], [2], [4], [5], [6], [7], [8], [9], [13], [18], [19], [20], [21], [22], [23], [24], [25], [26], [28], [29] and references therein. In particular, [9] describes a new setting for spectral estimation. This so-called THREE algorithm appears to allow for higher resolution in prescribed frequency bands and to be particularly suitable in case of short observation records. It effectively detects spectral lines and steep variations (see [36] for a recent biomedical application). An outline of this method is as follows. A given realization of a stochastic process (a finite collection of data $y_{1} \ldots y_{N}$ ) is fed to a suitably structured bank of filters, and the steady-state covariance matrix of the resulting output is estimated by statistical methods. Only zeroth-order covariance lags of the output of the filters need to be estimated, ensuring statistical robustness of the method. Finding now an input process whose rational spectrum is compatible with the estimated covariance poses naturally a Nevanlinna-Pick interpolation problem with bounded degree. The solution of this interpolation problem is considered as a mean of estimating the spectrum. A particular case described in the paper is the maximum differential entropy spectrum estimate, which amounts to the so-called central solution in the Nevanlinna-Pick theory. More generally, the scheme allows for a non constant a priori estimate $\Psi$ of the spectrum. The Byrnes-Georgiou-Lindquist school has shown how this and other important problems of control theory may be advantageously cast in the frame of convex optimization. These problems admit a finite dimensional dual (multipliers are matrices!) that can be shown to be solvable. The latter result, due to Byrnes and Lindquist [8] (see also [16]) is, however, nontrivial since the optimization occurs on an open, unbounded set of Hermitian matrices. The numerical solution of the dual problem is also challenging [9], [13], [35], since the gradient of the dual functional tends to infinity at the boundary of the feasible set. Finally, reparametrization of the problem may lead to loss of global concavity, see the discussion in [28, Section VII].

This paper adds to this effort in that we consider estimation of a multivariate spectral density in the spirit of THREE [9], but employing a different metric for the optimization part, namely the Hellinger distance as in [17]. In papers [6], [7], Byrnes, Gusev and Lindquist chose the Kullback-Leibler divergence as a frequency weighted entropy measure, thus introducing a broad generalization of Burg's maximum entropy method. More recently, this motivation was supported by the well-known connection with prediction error methods, see e.g. [41], [32]. In the multivariable case, a Kullback-Leibler pseudodistance may also be readily defined [24] inspired by the von Neumann's relative entropy [44], [43] of statistical quantum mechanics. The resulting spectrum approximation problem, however, leads to computable solutions of bounded McMillan degree only in the case when the prior spectral density is the identity matrix [24], [17] (maximum entropy solution). On the contrary, with a suitable extension of the scalar Hellinger distance introduced in [17], the Hellinger approximation generalizes nicely to the multivariable case for any prior estimate $\Psi$ of the spectrum.

The main contributions of this paper, after some background material in Sections II-IV, are found in Sections V-VIII. In Section $\mathrm{V}$, we establish strong convexity and smoothness of the dual functional on a certain domain of Hermitian matrices.

Work partially supported by the MIUR-PRIN Italian grant "Identification and Control of Industrial Systems"

A. Ferrante and F. Ramponi are with the Dipartimento di Ingegneria dell'Informazione, Università di Padova, via Gradenigo 6/B, 35131 Padova, Italy augusto@dei.unipd.it, rampo@dei.unipd.it

M. Pavon is with the Dipartimento di Matematica Pura ed Applicata, Università di Padova, via Trieste 63, 35131 Padova, Italy pavon@math . un ipd. it 
In Section VI, we analyze in detail a variant of a Newton-type matricial iteration designed to numerically solve the dual of the multivariable spectrum approximation problem. It had originally been sketched in [17]. The computational burden is dramatically reduced by systematically resorting to solutions of Lyapunov and Riccati equations thanks to various nontrivial results of spectral factorization. We then show in Section VII that the algorithm is globally convergent. Finally, in Section VIII, we present guidelines for its application to multivariate spectral estimation and present some simulations comparing to existing methods. Simulation in the multivariable case shows that, at the price of some moderate extra complexity in the model, our method may perform much better than MATLAB's PEM and MATLAB's N4SID in the case of a short observation record.

\section{CONSTRAINED SPECTRUM APPROXIMATION}

Paper [18] introduces and solves the following moment problem: Given a bank of filters described by an input-to-state stable transfer function $G(z)=(z I-A)^{-1} B$ and a state covariance matrix $\Sigma$, give necessary and sufficient conditions for the existence of input spectra $\Phi\left(\mathrm{e}^{\mathrm{j} \vartheta}\right)$ such that the steady state output has variance $\Sigma$, that is,

$$
\int G \Phi G^{*}=\Sigma .
$$

Moreover, parametrize the set of all such spectra (here, and in the sequel, integration takes place on the unit circle $\mathbb{T}$ with respect to normalized Lebesgue measure $d \vartheta / 2 \pi)$. Throughout this paper we use the following notations: $A^{*}=\bar{A}^{\top}$ for matrices and $G^{*} \equiv G^{*}(z)=G^{\top}\left(z^{-1}\right)$ for spectra and transfer functions. The scalar product between square matrices is defined as $\langle A, B\rangle=\operatorname{tr} A B^{*}$. Let $\mathcal{S}=\mathcal{S}_{+}^{m \times m}(\mathbb{T})$ be the family of $\mathbb{C}^{m \times m}$-valued functions defined on the unit circle which are Hermitian, positive-definite, bounded and coercive. We have the following existence result [18]: There exists $\Phi \in \mathcal{S}$ satisfying (1) if and only if there exists $H \in \mathbb{C}^{m \times n}$ such that

$$
\Sigma-A \Sigma A^{*}=B H+H^{*} B^{*}
$$

Paper [28] deals with the following (scalar) spectrum approximation problem: When constraint (1) is feasible, find the spectrum $\Phi$ which minimizes the Kullback-Leibler pseudo distance

$$
d_{K L}(\Psi, \Phi)=\int \Psi \log \frac{\Psi}{\Phi}
$$

from an "a priori" spectrum $\Psi$, subject to the constraint (1). It turns out that, if the prior $\Psi$ is rational, the solution is also rational, and with degree that can be bounded in terms of the degrees of $G(z)$ and $\Psi$. This problem again admits the maximum differential entropy spectrum (compatible with the constraint) as a particular case $\left(\Psi\left(\mathrm{e}^{\mathrm{j} \vartheta}\right) \equiv 1\right)$. The above minimization poses naturally a variational problem, which can be solved using Lagrange theory. Its dual problem admits a maximum and can be solved exploiting numerical algorithms. In [15] we restated and solved a similar variational problem with respect to a different metric, namely the Hellinger distance:

$$
d_{H}(\Psi, \Phi)=\sqrt{\int(\sqrt{\Psi}-\sqrt{\Phi})^{2}}
$$

Equation (3) defines a bona fide distance, well-known in mathematical statistics. The main advantage of this approach to spectral approximation is that it easily generalizes to the multivariable case, whereas log-like functionals do not enjoy this property [1], [22], [24], [17].

\section{FeASIBILITY AND THE OPERATOR $\Gamma$}

In this section, we discuss in depth the feasibility of [1]. Following [28] and [17], let $\mathcal{H}(n)=\left\{M \in \mathbb{C}^{n \times n}: M=\right.$ $\left.M^{*}\right\}$, let $\mathcal{C}(\mathbb{T} ; \mathcal{H}(m))$ be the space of $\mathcal{H}(m)$-valued continuous functions defined on the unit circle, and let the operator $\Gamma: \mathcal{C}(\mathbb{T} ; \mathcal{H}(m)) \rightarrow \mathcal{H}(n)$ be defined as follows:

$$
\Gamma(\Phi):=\int G \Phi G^{*}
$$

We are interested in the range of the operator $\Gamma$ which, having to deal with Hermitian matrices, we consider as a vector space over the reals.

Proposition 3.1: The following facts hold:

1) Let $\Sigma=\Sigma^{*}>0$. The following are equivalent:

- There exists $H \in \mathbb{C}^{m \times n}$ such that identity $(2)$ holds.

- There exists $\Phi \in \mathcal{S}_{+}^{m \times m}(\mathbb{T})$ such that $\int G \Phi G^{*}=\Sigma$.

- There exists $\Phi \in \mathcal{C}(\mathbb{T} ; \mathcal{H}(m)), \Phi>0$ such that $\Gamma(\Phi)=\Sigma$.

2) Let $\Sigma=\Sigma^{*}$ (not necessarily positive definite). There exists $H \in \mathbb{C}^{m \times n}$ such that identity 2 holds if and only if $\Sigma \in$ Range $\Gamma$.

3) $X \in$ Range $\Gamma^{\perp}$ if and only if $G^{*}\left(\mathrm{e}^{\mathrm{j} \vartheta}\right) X G\left(\mathrm{e}^{\mathrm{j} \vartheta}\right)=0 \forall \vartheta \in[0,2 \pi]$. 
Proof:

As stated above, it was proved in [18] that there exists $H \in \mathbb{C}^{m \times n}$ such that identity (2) holds with Hermitian and positive definite $\Sigma$ if and only if $\Sigma=\int G \Phi G^{*}$ for some $\Phi \in \mathcal{S}_{+}^{m \times m}(\mathbb{T}), \Phi>0$. A similar result, albeit with a different algebraic formulation of the feasibility condition, was proved in [17, Proposition 2.1]. The proof of Fact 1 is straightforward once we note that the "if" part of the proof of [17, Proposition 2.1] is constructive, and exhibits a continuous spectrum. Hence, the fact that there exists a spectrum $\Phi$ such that $\Sigma=\int G \Phi G^{*}$ is equivalent to there exists a continuous spectrum such that the same holds.

As for the second assertion, let $\Sigma \in \operatorname{Range} \Gamma$. Then there exists $\Phi \in \mathcal{C}(\mathbb{T} ; \mathcal{H}(m))$ such that

$$
\begin{aligned}
\Sigma & =\int G \Phi G^{*}=\int G\left(\Phi_{+}-\Phi_{-}\right) G^{*} \\
& =\int G \Phi_{+} G^{*}-\int G \Phi_{-} G^{*}=\Sigma_{+}-\Sigma_{-}
\end{aligned}
$$

where $\Phi_{+}$and $\Phi_{-}$are two spectra such that $\Phi_{+}-\Phi_{-}=\Phi$ (they can be chosen to be bounded away from zero) and where $\Sigma_{+}$ and $\Sigma_{-}$are symmetric positive definite. Hence $\Sigma$ is a difference of positive matrices for which (2) holds. This establishes (2) for $\Sigma$ itself. Vice versa, suppose that $(2)$ holds for an Hermitian $\Sigma$. Let $\Sigma_{\alpha}$ be the unique solution of the following Lyapunov equation:

$$
\Sigma_{\alpha}-A \Sigma_{\alpha} A^{*}=B\left(\alpha B^{*}\right)+\left(\alpha B^{*}\right)^{*} B^{*}=2 \alpha B B^{*}
$$

where $\alpha \in \mathbb{R}$. Then $\Sigma_{\alpha}$ depends linearly upon $\alpha$, i.e. $\Sigma_{\alpha}=\alpha \Sigma_{1}$, where $\Sigma_{1}>0$ since $(A, B)$ is reachable. Thus, there exists an $\alpha$ such that $\Sigma_{\alpha}>0$ and $\Sigma_{\alpha}>\Sigma$. Let $\Sigma_{-}=\Sigma_{\alpha}-\Sigma$. Then $\Sigma_{-}>0$, and since (2) holds for $\Sigma$ and $\Sigma_{\alpha}$, it also holds for $\Sigma_{-}$. Then assertion 1 implies that there exist $\Phi_{\alpha}>0$ and $\Phi_{2}>0$ in $\mathcal{C}(\mathbb{T} ; \mathcal{H}(m))$ such that $\Sigma_{\alpha}=\int G \Phi_{\alpha} G^{*}$ and $\Sigma_{-}=\int G \Phi_{2} G^{*}$, hence $\Sigma=\int G\left(\Phi_{\alpha}-\Phi_{2}\right) G^{*}$ and assertion 2 follows.

The third assertion is a simple geometrical fact: If $X \in \operatorname{Range} \Gamma^{\perp}$, then for any $\Phi \in \mathcal{C}(\mathbb{T} ; \mathcal{H}(m))$

$$
0=\left\langle X, \int G \Phi G^{*}\right\rangle=\operatorname{tr} X \int G \Phi G^{*}=\operatorname{tr} \int\left(G^{*} X G\right) \Phi
$$

and the conclusion follows.

Remark 3.2: The underlying statement in Proposition 3.1. Facts 1 and 2, is that if we defined $\Gamma$ over the vector space of finite linear combinations of functions in $\mathcal{S}_{+}^{m \times m}(\mathbb{T})$, its range would remain the same.

Remark 3.3: Proposition 3.1 shows that Range $\Gamma$ is the set of all the Hermitian matrices $\Sigma$ for which there exists $H$ such that (2) holds. This fact will be useful in numerical computations. Indeed, Range $\Gamma$ is obviously finite-dimensional, and if $\left\{H_{1}, \ldots, H_{N}\right\}$ is a base of $\mathbb{C}^{m \times n}$, then the corresponding solutions $\left\{\Sigma_{1}, \ldots, \Sigma_{N}\right\}$ of 2 , considered as a discrete-time Lyapunov equation in the unknown $\Sigma$, generate Range $\Gamma$. Note that $\left\{\Sigma_{1}, \ldots, \Sigma_{N}\right\}$ are not necessarily linearly independent.

\section{Multivariable SPECTRUm APPRoximation in the Hellinger distance}

Let the function $d_{H}: \mathcal{S}_{+}^{m \times m}(\mathbb{T}) \times \mathcal{S}_{+}^{m \times m}(\mathbb{T}) \rightarrow \mathbb{R}^{+}$be defined as follows:

$$
\begin{aligned}
d_{H}(\Psi, \Phi)^{2}:= & \inf _{W_{\Psi}, W_{\Phi}} \operatorname{tr} \int\left(W_{\Psi}-W_{\Phi}\right)\left(W_{\Psi}-W_{\Phi}\right)^{*} \\
\text { s. t. } & W_{\Psi} W_{\Psi}^{*}=\Psi, \quad W_{\Phi} W_{\Phi}^{*}=\Phi, \\
& W_{\Psi} \text { and } W_{\Phi} \text { of dimension } m \times m
\end{aligned}
$$

that is, $d_{H}(\Psi, \Phi)$ is the $L^{2}$ distance between the sets of square spectral factors of the two spectra. It was shown in [17] that the infimum in (5) is actually a minimum and that $d_{H}$ is a bona fide distance between spectral densities, and reduces to the ordinary Hellinger distance in the scalar case. It was also shown there that the minimum in (5) is the same if we fix a square spectral factor $W_{\Psi}$ of $\Psi$, and then minimize over the spectral factors of $\Phi$ :

$$
\begin{gathered}
d_{H}(\Psi, \Phi)^{2} \equiv \min _{W} \operatorname{tr} \int\left(W_{\Psi}-W\right)\left(W_{\Psi}-W\right)^{*} \\
\text { s. t. } W W^{*}=\Phi
\end{gathered}
$$

The multivariable spectrum approximation problem addressed in [17] is the following. Let $G(z)=(z I-A)^{-1} B$, where $A$ is stable, $B$ has full rank and $(A, B)$ is reachable. Given $\Psi \in \mathcal{S}$, find

$$
\arg \min _{\Phi \in \mathcal{S}} d_{H}(\Psi, \Phi) \text { s. t. } \int G \Phi G^{*}=\Sigma
$$


Since $\Sigma>0$, applying the following change of base to $G(z)$ :

$$
\begin{aligned}
\bar{A} & =\Sigma^{-1 / 2} A \Sigma^{1 / 2}, \\
\bar{B} & =\Sigma^{-1 / 2} B, \\
\bar{G}(z) & =(z I-\bar{A})^{-1} \bar{B}=\Sigma^{-1 / 2} G(z),
\end{aligned}
$$

it is easy to see that there is no loss of generality in taking $\Sigma=I$. Thus, once a factor $W_{\Psi}$ of $\Psi$ is fixed, (7) reduces to:

$$
\begin{aligned}
& \arg \min _{W} \operatorname{tr} \int\left(W_{\Psi}-W\right)\left(W_{\Psi}-W\right)^{*} \\
& \text { s. t. } \int G W W^{*} G^{*}=I
\end{aligned}
$$

which is an $L^{2}$ constrained minimization.

Remark 4.1: In [17, Theorem 6.1], it was shown that the minimizer in (6) is explicitly given by

$$
\hat{W}_{\Phi}=\Phi^{1 / 2}\left[\Phi^{1 / 2} \Psi \Phi^{1 / 2}\right]^{-1 / 2} \Phi^{1 / 2} W_{\Psi} .
$$

Nevertheless, to solve the approximation problem (8), we do not need to employ 9 (see below).

\section{A. Variational analysis}

Now let us assume that the problem is feasible, i.e., condition (2) holds. To solve (8), form the Lagrangian:

$$
\begin{aligned}
L(W, \Lambda) & =\operatorname{tr} \int\left(W_{\Psi}-W\right)\left(W_{\Psi}-W\right)^{*} \\
& +\left\langle\Lambda, \int G W W^{*} G^{*}-I\right\rangle
\end{aligned}
$$

where $\Lambda \in \mathcal{H}(n)$. Since $\int G W W^{*} G^{*} \in \operatorname{Range} \Gamma$ by construction, and $I \in$ Range $\Gamma$ by the feasibility assumption, it is natural, though not strictly necessary, to restrict a priori the Lagrange parameter $\Lambda$ to Range $\Gamma$ (a $\Lambda \in \operatorname{Range} \Gamma^{\perp}$ would not play any role in the above Lagrangian).

We proceed with unconstrained minimization of (10). The functional $(10)$ is convex and differentiable in $W$. Thus, to find the unique minimizing solution we impose that the first variation of $(10)$ is zero in each direction $\delta W$. We easily find the following condition for $W$ (see [17] for the details):

$$
W-W_{\Psi}+G^{*} \Lambda G W=0
$$

To carry on with the computations, and to ensure that the resulting optimum spectrum is integrable over the unit circle, we require a posteriori that $\Lambda$ belongs to the following set:

$$
\mathcal{L}^{H}=\left\{\Lambda \in \mathcal{H}(n), \quad I+G^{*} \Lambda G>0 \quad \forall \mathrm{e}^{\mathrm{j} \vartheta} \in \mathbb{T}\right\}
$$

that is, $\Lambda \in \mathcal{L}_{\Gamma}^{H}$, where

$$
\mathcal{L}_{\Gamma}^{H}:=\text { Range } \Gamma \cap \mathcal{L}^{H} .
$$

If this is the case, the optimal spectral factor and the corresponding optimal spectral density are easily found to be:

$$
\begin{aligned}
\hat{W} & =\left(I+G^{*} \Lambda G\right)^{-1} W_{\Psi}, \\
\hat{\Phi} & =\left(I+G^{*} \Lambda G\right)^{-1} \Psi\left(I+G^{*} \Lambda G\right)^{-1}
\end{aligned}
$$

Remark 4.2: Observe that, when $\Psi$ is rational, (13) yields a rational spectrum with McMillan degree that can be bounded. The same applies to scalar spectrum approximation problem in a Kullback-Leibler type distance where the degree of the optimal approximant is actually lower [28]. In the multivariable case, however, the Kullback-Leibler solution is computable and of bounded McMillan degree only when $\Psi=I$ (maximum entropy solution), see [22, Theorem 1], [24, Section 4].

Consider now the issue of existence of a matrix $\Lambda \in \mathcal{L}_{\Gamma}^{H}$ such that

$$
\int G\left(I+G^{*} \Lambda G\right)^{-1} \Psi\left(I+G^{*} \Lambda G\right)^{-1} G^{*}=I
$$

that is, such that the corresponding optimal spectrum satisfies the constraint (17. A key result of [17, Theorem 7.7], inspired by a fundamental result of Byrnes and Lindquist [8], states that such a $\Lambda$ indeed exists and is unique, therefore establishing that for such $\Lambda \sqrt{13}$ is the solution to problem $(8)$.

Remark 4.3: Identity (14) is attained at $\Lambda=0$ if and only if $\Psi$ itself satisfies the contraint (1). The "only if" part is trivial. As for the "if" part, let $\int G \Psi G^{*}=I$. Then substituting $\Lambda=0$ into $\sqrt{13}$ we obtain the spectrum $\hat{\Phi} \equiv \Psi$, which has the least 
possible distance $d_{H}=0$ from $\Psi$ and hence is automatically optimal, and trivially satisfies (14). The assertion follows from the uniqueness of $\Lambda$.

In order to find the optimal $\Lambda$, we form the dual functional (see [17]):

$$
L_{d}(\Lambda)=L(\hat{W}, \Lambda)=\operatorname{tr} \int\left(\Psi-\left(I+G^{*} \Lambda G\right)^{-1} \Psi\right)-\operatorname{tr} \Lambda
$$

Notice that $L_{d}(\Lambda)$ is finite on $\mathcal{L}_{\Gamma}^{H}$. Recall that the dual of a Lagrangian functional is always concave and that a finite convex (or concave) function defined on a finite dimensional space is continuous on the interior of its domain (see [31] or [37]). Instead of maximizing (15), we consider the equivalent problem of minimizing the following functional:

$$
J_{\Psi}(\Lambda)=-L_{d}(\Lambda)+\operatorname{tr} \int \Psi=\operatorname{tr} \int\left(I+G^{*} \Lambda G\right)^{-1} \Psi+\operatorname{tr} \Lambda
$$

The minimization of the convex and continuous functional $J_{\Psi}$ over $\mathcal{L}_{\Gamma}^{H}$ is the main subject of this paper. The following sections are dedicated to prove strict convexity and smoothness of $J_{\Psi}$, to describe a Newton-type algorithm for its numerical minimization, and to prove the global convergence of that algorithm. Some numerical simulations follow.

\section{PRoperties of the Functional $J_{\Psi}$}

In this section, we establish various properties of the functional $J_{\Psi}$ on $\mathcal{L}_{\Gamma}^{H}$. We begin by recalling a few basic definitions and facts from multivariate analysis. A function $f: S \subset \mathbb{R}^{N} \rightarrow \mathbb{R}^{M}$ is (Fréchet) differentiable on the open set $S$ if for all $x \in S$ there exists a linear map $L_{x}: \mathbb{R}^{N} \rightarrow \mathbb{R}^{M}$ such that

$$
\lim _{h \rightarrow 0} \frac{\left\|f(x+h)-f(x)-L_{x}(h)\right\|}{\|h\|}=0 .
$$

A function $f$ is said to be $C^{0}(S)$ if it is continuous on $S$. Also, $f$ is said to be $C^{1}(S)$ if it is differentiable at each $x \in S$ and if the operator $D f$ defined by

$$
D f(x):=L_{x}
$$

is $C^{0}(S)$. Now the derivative $D f: S \rightarrow L\left(\mathbb{R}^{N}, \mathbb{R}^{M}\right) \simeq \mathbb{R}^{M N}$ is itself a function between finite-dimensional spaces. If $D f$ is $C^{1}(S)$, then $f$ is said to be $C^{2}(S)$. Proceeding in this way, the $C^{k}$-differentiability of $f$ can be defined. Finally, $f$ is said to be of class $C^{\infty}(S)$ if it is $C^{k}(S)$ for all $k \in \mathbb{N}$. A standard result in analysis states that $f \in C^{1}(S)$ if and only if the partial derivatives $\frac{\partial f_{m}}{\partial x_{n}}$ (where $f_{m}$ is the $m$-th component of $f$ ) exist and are continuous on $S$ (see for instance [38, Theorem 9.21]). It follows that $f \in C^{k}(S)$ if and only if $f$ has in $S$ continuous partial derivatives of any order up to $k$, that is:

$$
\frac{\partial^{h} f_{m}}{\partial x_{n_{1}} \cdots \partial x_{n_{h}}} \in C^{0}(S)
$$

for all $m, n_{i}, h$ s.t. $1 \leq m \leq M, 1 \leq n_{i} \leq N$, and $0 \leq h \leq k$.

In our setting, where $J_{\Psi}: \mathcal{L}_{\Gamma}^{H} \subset$ Range $\Gamma \rightarrow \mathbb{R}$, the role of the above partial derivatives is played by the directional (or Gâteaux) derivatives,

$$
\delta^{h} J_{\Psi}\left(\Lambda ; \overline{\left.\delta \Lambda_{n_{1}}, \ldots, \delta \bar{\Lambda}_{n_{h}}\right)}\right.
$$

where $1 \leq n_{i} \leq d$ and $\left\{\overline{\delta \Lambda}_{1}, \ldots, \overline{\delta \Lambda_{d}}\right\}$ is a fixed orthonormal base of Range $\Gamma$. A fortiori, if we show that $J_{\Psi}$ has on $\mathcal{L}_{\Gamma}^{H}$ continuous directional derivatives of any order up to $k$, taken in whatever directions $\left\{\delta \Lambda_{1}, \ldots, \delta \Lambda_{k}\right\} \subset$ Range $\Gamma$, then we can say that $J_{\Psi} \in C^{k}\left(\mathcal{L}_{\Gamma}^{H}\right)$.

Lemma 5.1: Let $H \in \mathcal{H}(n)$ and $m$ be its minimum eigenvalue. The map $H \mapsto m$ is continuous.

Proof:

The map from a matrix $H$ to the vector of coefficients of its characteristic polynomial $a(s)=\operatorname{det}(s I-H)=a_{0}+\ldots+$ $a_{n-1} s^{n-1}+s^{n}$ is continuous. Indeed, each of the coefficients of $a(s)$ is obtained by means of sums and products of elements of $H$. Moreover, it is a well-known fact (see for example [34]) that the mapping from the coefficients of a monic polynomial to its roots is continuous, in the following sense: Given $a(s)=s^{n}+\sum_{i=0}^{n-1} a_{i} s^{i}$, let $\lambda_{i}$ be the zeros of $a(s)$ and $\nu_{i}$ the respective multiplicities. For all $\varepsilon>0$, there exists $\delta>0$ such that if $b(s)=s^{n}+\sum_{i=0}^{n-1} b_{i} s^{i}$ and $\left|b_{i}-a_{i}\right|<\delta$ for all $i=0,1, \ldots, n-1$, then $b(s)$ has $\nu_{i}$ zeros in the ball centered in $\lambda_{i}$ with radius $\varepsilon$. In conclusion, if $H$ is Hermitian, the mapping from $H$ to its minimum (real) eigenvalue is continuous.

Lemma 5.2: Define $Q_{\Lambda}(z)=I+G^{*}(z) \Lambda G(z)$. Consider a sequence $\Lambda_{n} \in \mathcal{L}_{\Gamma}^{H}$ converging to $\Lambda \in \mathcal{L}_{\Gamma}^{H}$. Then $Q_{\Lambda_{n}}^{-1}$ are well defined and continuous on $\mathbb{T}$ and converge uniformly to $Q_{\Lambda}^{-1}$ on $\mathbb{T}$.

Proof: 
Observe that, for $\Lambda \in \mathcal{L}_{\Gamma}^{H}, Q_{\Lambda}$ is a positive definite, continuous matrix function on $\mathbb{T}$. By Lemma 5.1 there exists a continuous function $m_{\Lambda}\left(\mathrm{e}^{\mathrm{j} \vartheta}\right)>0$ such that $Q_{\Lambda}\left(\mathrm{e}^{\mathrm{j} \vartheta}\right) \geq m_{\Lambda}\left(\mathrm{e}^{\mathrm{j} \vartheta}\right) I$ for each $\vartheta$. Hence, $Q_{\Lambda}\left(\mathrm{e}^{\mathrm{j} \vartheta}\right) \geq m_{\Lambda} I, \forall \vartheta$, where $m_{\Lambda}:=$ $\min _{\vartheta} m_{\Lambda}\left(\mathrm{e}^{\mathrm{j} \vartheta}\right)>0$. Let $\delta \Lambda \in B(0, \varepsilon)$, the closed ball of radius $\varepsilon$ centered in 0 . Now,

$$
\left\|G^{*}\left(\mathrm{e}^{\mathrm{j} \vartheta}\right) \delta \Lambda G\left(\mathrm{e}^{\mathrm{j} \vartheta}\right)\right\| \leq\|\delta \Lambda\| M_{G} \leq \varepsilon M_{G}
$$

where

$$
M_{G}=\max _{\vartheta}\left\|G^{*}\left(\mathrm{e}^{\mathrm{j} \vartheta}\right)\right\|\left\|G\left(\mathrm{e}^{\mathrm{j} \vartheta}\right)\right\| .
$$

Thus, if we choose $\varepsilon<m_{\Lambda} / M_{G}$, then $\left\|G^{*}\left(\mathrm{e}^{\mathrm{j} \vartheta}\right) \delta \Lambda G\left(\mathrm{e}^{\mathrm{j} \vartheta}\right)\right\|<m_{\Lambda}$. Hence, $I+G^{*}(\Lambda+\delta \Lambda) G$ describes, as $(\delta \Lambda, \vartheta)$ varies in $B(0, \varepsilon) \times[-\pi, \pi]$, a compact set that does not contain any singular matrix. Now recall that the matrix inversion operator is continuous at any nonsingular matrix. Hence, $Q_{\Lambda+\delta \Lambda}^{-1}\left(\mathrm{e}^{\mathrm{j} \vartheta}\right)$ admits a uniform bound $M(\Lambda, \epsilon)$ on $B(0, \varepsilon) \times[-\pi, \pi]$. Since $\Lambda_{n} \rightarrow \Lambda$, for $n$ sufficiently large, $\left(\Lambda_{n}-\Lambda\right) \in B(0, \varepsilon)$. Then

$$
\begin{aligned}
\sup _{\vartheta}\left\|Q_{\Lambda_{n}}^{-1}-Q_{\Lambda}^{-1}\right\| & =\sup _{\vartheta}\left\|Q_{\Lambda_{n}}^{-1}\left[G^{*}\left(\Lambda-\Lambda_{n}\right) G\right] Q_{\Lambda}^{-1}\right\| \\
& \leq M^{2} \sup _{\vartheta}\left\|G^{*}\left(\Lambda-\Lambda_{n}\right) G\right\| \\
& \leq M^{2} \varepsilon M_{G} .
\end{aligned}
$$

This implies that $Q_{\Lambda_{n}}^{-1} \rightarrow Q_{\Lambda}^{-1}$ uniformly on $\mathbb{T}$.

Theorem 5.3: Consider $J_{\Psi}: \mathcal{L}_{\Gamma}^{H} \subset$ Range $\Gamma \rightarrow \mathbb{R}$. Then

1) $J_{\Psi} \in C^{\infty}\left(\mathcal{L}_{\Gamma}^{H}\right)$.

2) $J_{\Psi}$ is strictly convex on $\mathcal{L}_{\Gamma}^{H}$.

Proof:

Let $\mathcal{I}: A \mapsto A^{-1}$ be the matrix inversion operator. Making use of

$$
\delta \mathcal{I}(A ; \delta A)=-A^{-1} \delta A A^{-1},
$$

the first variation of $J_{\Psi}(\Lambda)$ in an arbitrary direction $\delta \Lambda_{1}$ is found to be:

$$
\begin{aligned}
\delta J_{\Psi}\left(\Lambda ; \delta \Lambda_{1}\right) & =-\operatorname{tr} \int Q_{\Lambda}^{-1} G^{*} \delta \Lambda_{1} G Q_{\Lambda}^{-1} \Psi+\operatorname{tr} \delta \Lambda_{1} \\
& =\left\langle I-\int G Q_{\Lambda}^{-1} \Psi Q_{\Lambda}^{-1} G^{*}, \quad \delta \Lambda_{1}\right\rangle
\end{aligned}
$$

The linear functional $\nabla J_{\Psi, \Lambda}(\cdot):=\delta J_{\Psi}(\Lambda ; \cdot)$ defined by $(18)$ is the gradient of $J_{\Psi}$ at $\Lambda$. To prove that $J_{\Psi} \in C^{1}\left(\mathcal{L}_{\Gamma}^{H}\right)$ we must show that, for any fixed $\delta \Lambda_{1}, \delta J_{\Psi}\left(\Lambda ; \delta \Lambda_{1}\right)$ is continuous in the variable $\Lambda$ (it follows that $\nabla J_{\Psi, \Lambda}(\cdot)$ is also continuous in $\Lambda$ ). Consider a sequence $M_{n} \in$ Range $\Gamma$ converging to 0 . By Lemma 5.2, $Q_{\Lambda+M_{n}}^{-1}$ converge uniformly to $Q_{\Lambda}^{-1}$. Recall that $\Psi$ is bounded. Applying elementwise the bounded convergence theorem, we get

$$
\lim _{n \rightarrow+\infty} \int Q_{\Lambda+M_{n}}^{-1} G^{*} \delta \Lambda_{1} G Q_{\Lambda+M_{n}}^{-1} \Psi=\int Q_{\Lambda}^{-1} G^{*} \delta \Lambda_{1} G Q_{\Lambda}^{-1} \Psi
$$

Hence, for all $\delta \Lambda_{1} \in \operatorname{Range} \Gamma, \delta J_{\Psi}\left(\Lambda ; \delta \Lambda_{1}\right)$ is continuous, i.e. $J_{\Psi} \in C^{1}\left(\mathcal{L}_{\Gamma}^{H}\right)$. The second variation of $J_{\Psi}$, say in direction $\delta \Lambda_{2}$, is easily obtained applying (17) and the chain rule to 18 :

$$
\begin{aligned}
& \delta^{2} J_{\Psi}\left(\Lambda ; \delta \Lambda_{1}, \delta \Lambda_{2}\right) \\
& =\operatorname{tr} \int W_{\Psi}^{*} Q_{\Lambda}^{-1} G^{*} \delta \Lambda_{2} G Q_{\Lambda}^{-1} G^{*} \delta \Lambda_{1} G Q_{\Lambda}^{-1} W_{\Psi} \\
& +\operatorname{tr} \int W_{\Psi}^{*} Q_{\Lambda}^{-1} G^{*} \delta \Lambda_{1} G Q_{\Lambda}^{-1} G^{*} \delta \Lambda_{2} G Q_{\Lambda}^{-1} W_{\Psi}
\end{aligned}
$$

The bilinear form $H_{\Lambda}(\cdot, \cdot):=\delta^{2} J_{\Psi}(\Lambda ; \cdot, \cdot)$ is the Hessian of $J_{\Psi}$ at $\Lambda$. Again, continuity of $\delta^{2} J_{\Psi}\left(\Lambda ; \delta \Lambda_{1}, \delta \Lambda_{2}\right)$ can be established by the previous argument in view of Lemma 5.2 Similarly, it can be shown that $J_{\Psi}$ has continuous directional derivatives of any order. Thus $J_{\Psi} \in C^{k}\left(\mathcal{L}_{\Gamma}^{H}\right)$ for any $k$, and the first assertion follows. Finally, we show that $J_{\Psi}$ is strictly convex on $\mathcal{L}_{\Gamma}^{H}$. A standard result in the theory of convex functions states that if a function $f: S \subset \mathbb{R}^{N} \rightarrow \mathbb{R}$ is $C^{2}(S)$ (where $S$ is open), then $f$ is strictly convex on $S$ if and only if its Hessian $H_{x}$ is positive definite at each $x \in S$. Consider $H_{\Lambda}(\delta \Lambda, \delta \Lambda)=\delta^{2} J_{\Psi}(\Lambda ; \delta \Lambda, \delta \Lambda)$ for $\delta \Lambda \in$ Range $\Gamma \backslash\{0\}$. Since the integrand in $[19\}$ is positive semidefinite, it follows that $H_{\Lambda}(\delta \Lambda, \delta \Lambda) \geq 0$. In view of Point 3 in Proposition 3.1 the integrand is not identically zero and $H_{\Lambda}(\delta \Lambda, \delta \Lambda)>0$. It follows that $J_{\Psi}$ is strictly convex.

Loosely speaking, [17] establishes existence of the minimum by showing that $J_{\Psi}$ is bounded from below and that $J_{\Psi}(\Lambda) \rightarrow$ $+\infty$ whenever $\|\Lambda\| \rightarrow+\infty$ or $\Lambda$ approaches $\partial \mathcal{L}_{\Gamma}^{H}$, the boundary of $\mathcal{L}_{\Gamma}^{H}$. Since the minimum point cannot reside at infinity 
or at the boundary, we can then restrict the minimization problem to a sublevel set of $J_{\Psi}$. It follows from the continuity of $J_{\Psi}$ that such a set is compact, and the existence result follows from Weierstrass' theorem. Since $J_{\Psi}$ is strictly convex, the minimum point is unique.

\section{Vi. A matricial NeWton ALgorithm}

\section{A. Description of the iterative method}

The Newton algorithm is an iterative procedure for the search of roots of a function or the minimization of a functional. With respect to the latter objective, it can be formulated as follows. Let $f: S \rightarrow \mathbb{R}$ be a functional defined over $S \subset \mathbb{R}^{n}$. In order to find an estimate $\hat{x}$ of a minimum point $x^{*}$ of $f$,

1) Make an initial guess $x_{0}$, possibly near the minimum point.

2) At each iteration, compute the Newton step

$$
\Delta x_{i}=-H_{x_{i}}^{-1} \nabla f_{x_{i}}
$$

where $H_{x_{i}}$ is the Hessian of $f$ at $x_{i}$ and $\nabla f_{x_{i}}$ is the gradient of $f$ at $x_{i}$ (understood as a column vector).

3) Set $t_{i}^{0}=1$, and let $t_{i}^{k+1}=t_{i}^{k} / 2$ until both of the following conditions hold:

$$
\begin{gathered}
x_{i}+t_{i}^{k} \Delta x_{i} \in S \\
f\left(x_{i}+t_{i}^{k} \Delta x_{i}\right)<f\left(x_{i}\right)+\alpha t_{i}^{k} \nabla f_{x_{i}}^{\top} \Delta x_{i}
\end{gathered}
$$

where $\alpha$ is a real constant, $0<\alpha<1 / 2$.

4) Set $x_{i+1}=x_{i}+t_{i}^{k} \Delta x_{i}$.

5) Repeat steps 2, 3 and 4 until $\left|\nabla f_{x_{i}}\right|<\varepsilon$, where $\varepsilon$ is a (small) tolerance threshold, then set $\hat{x}=x_{i}$.

In its "pure" form, the iteration of the Newton algorithm only consists in step 2, which is indeed its essential part. Step 3 is the so-called backtracking procedure. For small $t$, if $f$ is sufficiently regular, we have $f\left(x_{i}+t \Delta x_{i}\right) \simeq f\left(x_{i}\right)+t \nabla f_{x_{i}}^{\top} \Delta x_{i}$. Since $\nabla f_{x_{i}}^{\top} \Delta x_{i}=-\nabla f_{x_{i}}^{\top} H_{x_{i}}^{-1} \nabla f_{x_{i}}<0$, condition 22) must hold for small $t$, hence step 3 must terminate at some iteration. Since $\nabla f_{x_{i}}^{\top} \Delta x_{i}<0,22$ implies $f\left(x_{i}+t_{i}^{k} \Delta x_{i}\right)<f\left(x_{i}\right)$. That is, $\left\{f\left(x_{i}\right)\right\}$ is a strictly decreasing sequence.

In essence, the "pure" Newton algorithm works very well when the starting point happens to be near the minimum and the function $f$ is there effectively approximated by a quadratic form, but it can suffer from numerical problems when this is not the case. The backtracking line search is a remedy to this drawback; moreover it can be shown that, under certain regularity assuptions on $f$, which hold in our case (see Section VIIT), after a finite number of iterations step 3 always selects the multiplier $t=1$, that is, the full step. During the latter stage, the convergence to the minimizing solution is quadratic, meaning that there exists a constant $C$ such that $\left\|x_{i+1}-x^{*}|| \leq C|| x_{i}-x^{*}\right\|^{2}$. This rate of convergence makes the Newton algorithm often preferable over other minimization methods (see [10]). We must minimize the functional $J_{\Psi}(\Lambda)$ over the set $\mathcal{L}_{\Gamma}^{H}$. As initial condition, we can safely choose 0 . Hence, set

$$
\Lambda_{0}=0
$$

It turns out that, although the problem is finite-dimensional, the inversion of the Hessian is more demanding than inverting a matrix. In order to compute the Newton step $\Delta \Lambda_{i}$, we must solve at $\Lambda_{i}$ the following linear equation:

$$
H_{\Lambda_{i}}\left(\Delta \Lambda_{i}, \cdot\right)=-\nabla J_{\Psi, \Lambda_{i}}(\cdot)
$$

where, once fixed $\Lambda_{i}, \nabla J_{\Psi, \Lambda_{i}}(\cdot)$ and $H_{\Lambda_{i}}(\cdot, \cdot)$ must be understood as a linear and a bilinear form, defined by (18) and (19) respectively. Comparing with the above definitions, 24 reduces to:

$$
\begin{aligned}
& \int G Q_{\Lambda_{i}}^{-1}\left[\left(G^{*}\left(\Delta \Lambda_{i}\right) G Q_{\Lambda_{i}}^{-1} \Psi\right)+\left(G^{*}\left(\Delta \Lambda_{i}\right) G Q_{\Lambda_{i}}^{-1} \Psi\right)^{*}\right] Q_{\Lambda_{i}}^{-1} G^{*} \\
& =\int G Q_{\Lambda_{i}}^{-1} \Psi Q_{\Lambda_{i}}^{-1} G^{*}-I
\end{aligned}
$$

In principle, equation (25) is not difficult to solve. We suggest the following procedure:

- At the beginning of the procedure, take a base $\left\{H_{1}, \ldots, H_{k}, \ldots, H_{N}\right\}$ of $\mathbb{C}^{m \times n}$. 1 Then compute the solutions $\left\{\Sigma_{1}, \ldots, \Sigma_{k}, \ldots, \Sigma_{N}\right\}$ of the following discrete-time Lyapunov equations:

$$
\Sigma_{k}-A \Sigma_{k} A^{*}=B H_{k}+H_{k}^{*} B^{*}
$$

As shown before, these solutions generate Range $\Gamma$.

- To compute $\Delta \Lambda_{i}$ at each step,

${ }^{1}$ Actually, it suffices to take the $\left\{H_{k}\right\}$ to be a base of $\mathbb{C}^{m \times n} \ominus \operatorname{Ker} R$, where the map $R$ is defined by

$$
R: H \mapsto B H+H^{*} B^{*} .
$$


1) Compute the integral

$$
Y=\int G Q_{\Lambda_{i}}^{-1} \Psi Q_{\Lambda_{i}}^{-1} G^{*}-I
$$

2) For each $\Sigma_{k}$ in the precomputed generators, compute the following integral:

$$
\begin{aligned}
Y_{k}=\int & G Q_{\Lambda_{i}}^{-1}\left[\left(G^{*} \Sigma_{k} G Q_{\Lambda_{i}}^{-1} \Psi\right)\right. \\
& \left.+\left(G^{*} \Sigma_{k} G Q_{\Lambda_{i}}^{-1} \Psi\right)^{*}\right] Q_{\Lambda_{i}}^{-1} G^{*}
\end{aligned}
$$

3) Solve, by means of linear algebraic methods (the Moore-Penrose pseudoinverse), the equation

$$
\sum_{k} \alpha_{k} Y_{k}=Y
$$

4) By linearity, the solution to 25 is

$$
\Delta \Lambda_{i}=\sum_{k} \alpha_{k} \Sigma_{k}
$$

It is clear that the real difficulty here is the computation of the integrals $(26)$ and 27$)$. This task requires extensive use of the following results of linear stochastic systems theory.

Lemma 6.1: Let $A$ be a stability matrix and $W(z)=C(z I-A)^{-1} B+D$ a minimal realization of a spectral factor of $\Phi(z)$. Let $\Pi$ be the unique solution to the Lyapunov equation

$$
\Pi=A \Pi A^{*}+B B^{*}
$$

Then the following hold:

1) $\int_{-\pi}^{\pi} \Phi\left(\mathrm{e}^{\mathrm{j} \vartheta}\right) \frac{\mathrm{d} \vartheta}{2 \pi}=C \Pi C^{*}+D D^{*}$

2) $Z(z)=C(z I-A)^{-1}\left(A \Pi C^{*}+B D^{*}\right)+\frac{1}{2}\left(C \Pi C^{*}+D D^{*}\right)$ is a realization of the causal part of $\Phi(z)$; that is, $Z(z)$ is analytic outside the unit circle and $\Phi(z)=Z(z)+Z^{*}(z)$.

Lemma 6.2: Let $Z(z)=C(z I-A)^{-1} G+\frac{1}{2} \Sigma$ be a minimal realization of the causal part of a spectrum $\Phi(z)$. Let $P_{-}$be the stabilizing solution of the following Algebraic Riccati Equation (ARE):

$$
P=A P A^{*}+\left(G-A P C^{*}\right)\left(\Sigma-C P C^{*}\right)^{-1}\left(G^{*}-C P A^{*}\right)
$$

Let moreover $D=\left(\Sigma-C P_{-} C^{*}\right)^{1 / 2}$ and $B=\left(G-A P_{-} C^{*}\right) D^{-1}$. Then $W(z)=C(z I-A)^{-1} B+D$ is the minimum phase spectral factor of $\Phi(z)$; that is, $W(z)$ is stable and with stable causal inverse, and $\Phi(z)=W(z) W^{*}(z)$.

Lemma 6.3: Let $F(z)=C(z I-A)^{-1} B+D$ be a square transfer function, where $D$ is invertible. Then

$$
F^{-1}(z)=-D^{-1} C\left(z I-\left(A-B D^{-1} C\right)\right)^{-1} B D^{-1}+D^{-1}
$$

is a realization of its inverse.

Lemma 6.4: For all matrices $P=P^{*} \in \mathbb{C}^{n \times n}$ the following identity holds:

$$
\begin{aligned}
& {\left[\begin{array}{cc}
B^{*}\left(z^{-1} I-A^{*}\right)^{-1} & I
\end{array}\right]\left[\begin{array}{cc}
A^{*} P A-P & A^{*} P B \\
B^{*} P A & B^{*} P B
\end{array}\right] \times} \\
& \times\left[\begin{array}{c}
(z I-A)^{-1} B \\
I
\end{array}\right]=0
\end{aligned}
$$

Lemma 6.5: Let $A$ be a stability matrix and $H(z)=C(z I-A)^{-1} B+D$ be a minimal realization. Let $P$ be the solution of the Lyapunov equation

$$
P=A^{*} P A+C^{*} C
$$

Let $\left[\begin{array}{c}K \\ J\end{array}\right]$ be an ortho-normal basis of $\operatorname{ker}\left[\begin{array}{ll}A^{*} P^{1 / 2} & C^{*}\end{array}\right]$ i.e.

$$
\left[\begin{array}{ll}
A^{*} P^{1 / 2} & C^{*}
\end{array}\right]\left[\begin{array}{c}
K \\
J
\end{array}\right]=0, \quad\left[\begin{array}{ll}
K^{*} & J^{*}
\end{array}\right]\left[\begin{array}{c}
K \\
J
\end{array}\right]=I .
$$

Let $G:=P^{-1 / 2} K$ and

$$
H_{1}(z):=\left(D^{*} C+B^{*} P A\right)(z I-A)^{-1} G+B^{*} P G+D^{*} J .
$$

Then, $H^{*}(z) H(z)=H_{1}(z) H_{1}^{*}(z)$.

Lemmas 6.1, 6.2 and 6.3 are standard results (see for example [14]). The proofs of Lemmas 6.4 and 6.5 can be found in [12, Appendix A] and [17, Appendix A], respectively. 
Remark 6.6: Lemma 6.5 not only gives us a tool to compute a left factor from a right factor of a given spectrum. It also works in the opposite direction. Indeed, let $W(z)=C(z I-A)^{-1} B+D$ be a minimal realization, and let $\zeta=z^{-1}$. Then

$$
\begin{aligned}
& \Phi(z)=W(z) W^{*}(z) \\
& =\left(C(z I-A)^{-1} B+D\right)\left(B^{\top}\left(z^{-1} I-A^{\top}\right)^{-1} C^{\top}+D^{\top}\right) \\
& =\left(B^{\top}\left(\zeta^{-1} I-A^{\top}\right)^{-1} C^{\top}+D^{\top}\right)^{\top}\left(B^{\top}\left(\zeta I-A^{\top}\right)^{-1} C^{\top}+D^{\top}\right) \\
& =\left(B^{\top}\left(\zeta I-A^{\top}\right)^{-1} C^{\top}+D^{\top}\right)^{*}\left(B^{\top}\left(\zeta I-A^{\top}\right)^{-1} C^{\top}+D^{\top}\right) \\
& :=H^{*}(\zeta) H(\zeta)
\end{aligned}
$$

Applying Lemma 6.5 we can find an $H_{1}(\zeta)=H(\zeta I-F)^{-1} G+K$ such that $H^{*}(\zeta) H(\zeta)=H_{1}(\zeta) H_{1}^{*}(\zeta)$. Now turning back to $z$ :

$$
\begin{aligned}
& H_{1}(\zeta) H_{1}^{*}(\zeta) \\
& =\left(H(\zeta I-F)^{-1} G+K\right)\left(G^{\top}\left(\zeta^{-1} I-F^{\top}\right)^{-1} H^{\top}+K^{\top}\right) \\
& =\left(G^{\top}\left(z^{-1} I-F^{\top}\right)^{-1} H^{\top}+K^{\top}\right)^{\top}\left(G^{\top}\left(z I-F^{\top}\right)^{-1} H^{\top}+K^{\top}\right) \\
& =\left(G^{\top}\left(z I-F^{\top}\right)^{-1} H^{\top}+K^{\top}\right)^{*}\left(G^{\top}\left(z I-F^{\top}\right)^{-1} H^{\top}+K^{\top}\right) \\
& =W_{1}^{*}(z) W_{1}(z)=\Phi(z)
\end{aligned}
$$

\section{B. Factorization of $Q_{\Lambda}^{-1}(z)$}

The first problem to solve is to obtain a spectral factor of $Q_{\Lambda}^{-1}(z)$, where $Q_{\Lambda}(z)=\left(I+G^{*}(z) \Lambda G(z)\right)$. To this end, note that

$$
\begin{aligned}
Q_{\Lambda}(z) & =\left[\begin{array}{cc}
B^{*}\left(z^{-1} I-A^{*}\right)^{-1} & I
\end{array}\right]\left[\begin{array}{cc}
\Lambda & 0 \\
0 & I
\end{array}\right] \times \\
& \times\left[\begin{array}{c}
(z I-A)^{-1} B \\
I
\end{array}\right]
\end{aligned}
$$

Applying lemma 6.4, we can rewrite 37] as

$$
\begin{aligned}
& Q_{\Lambda}(z)=\left[\begin{array}{ll}
B^{*}\left(z^{-1} I-A^{*}\right)^{-1} & I
\end{array}\right] \times \\
& \times\left[\begin{array}{cc}
A^{*} P A-P+\Lambda & A^{*} P B \\
B^{*} P A & B^{*} P B+I
\end{array}\right] \times \\
& \times\left[\begin{array}{c}
(z I-A)^{-1} B \\
I
\end{array}\right]
\end{aligned}
$$

Now, the following linear matrix inequality:

$$
\begin{aligned}
& {\left[\begin{array}{cc}
A^{*} P A-P+\Lambda & A^{*} P B \\
B^{*} P A & B^{*} P B+I
\end{array}\right]} \\
& =\left[\begin{array}{c}
M^{*} \\
N^{*}
\end{array}\right]\left[\begin{array}{ll}
M & N
\end{array}\right] \geq 0
\end{aligned}
$$

is solvable for $P=P^{*}>0$ if and only if such is the following ARE:

$$
P=A^{*} P A-A^{*} P B\left(B^{*} P B+I\right)^{-1} B^{*} P A+\Lambda
$$

The stabilizing solution $P$ of 40 gives a realization for the square, minimum phase co-analytic spectral factor of $Q(z)$. We have:

$$
\begin{aligned}
N & =N^{*}=\left(B^{*} P B+I\right)^{1 / 2} \\
M & =\left(B^{*} P B+I\right)^{-1 / 2} B^{*} P A \\
\Delta_{\Lambda}(z) & =\left[\begin{array}{ll}
M & N
\end{array}\right]\left[\begin{array}{c}
(z I-A)^{-1} B \\
I
\end{array}\right] \\
& =\left(B^{*} P B+I\right)^{-1 / 2} B^{*} P A(z I-A)^{-1} B \\
& +\left(B^{*} P B+I\right)^{1 / 2} \\
Q_{\Lambda}(z) & =\Delta_{\Lambda}^{*}(z) \Delta_{\Lambda}(z)
\end{aligned}
$$

and finally $Q_{\Lambda}^{-1}(z)=\Delta_{\Lambda}{ }^{-1}(z) \Delta_{\Lambda}{ }^{-*}(z)$ where, by means of lemma 6.3

$$
\begin{gathered}
\Delta_{\Lambda}{ }^{-1}(z)=-\left(B^{*} P B+I\right)^{-1} B^{*} P A(z I-\Gamma)^{-1} B \times \\
\times\left(B^{*} P B+I\right)^{-1 / 2}+\left(B^{*} P B+I\right)^{-1 / 2} \\
\Gamma=A-B\left(B^{*} P B+I\right)^{-1} B^{*} P A
\end{gathered}
$$


C. Computation of the integrals in (26) and 27)

By virtue of Lemma 6.5 and Remark 6.6, we can switch from a right factorization of a spectrum $\left(\Phi=H^{*} H\right)$ to a left factorization $\left(\Phi=W W^{*}\right)$, and vice versa. We will now show that both (26) and 27) can be reduced to integrals of the form

$$
\int G(z) \Delta_{\Lambda}^{-1}(z) \Phi(z) \Delta_{\Lambda}^{-*}(z) G^{*}(z)
$$

where $\Phi(z)$ is a spectrum. Indeed, let $\Phi_{\Psi}(z)=\Delta_{\Lambda}{ }^{-*}(z) \Psi(z) \Delta_{\Lambda}{ }^{-1}(z)$. Then

$$
\begin{aligned}
& \int G(z) Q_{\Lambda}^{-1}(z) \Psi(z) Q_{\Lambda}^{-1}(z) G^{*}(z) \\
& =\int G(z) \Delta_{\Lambda}{ }^{-1}(z)\left(\Delta_{\Lambda}{ }^{-*}(z) \Psi(z) \Delta_{\Lambda}{ }^{-1}(z)\right) \Delta_{\Lambda}{ }^{-*}(z) G^{*}(z)
\end{aligned}
$$

which has the form 43 with $\Phi=\Phi_{\Psi}$. Applying Lemma 6.5 we obtain a (left) spectral factor of $\Phi_{\Psi}(z)$ :

$$
\begin{aligned}
\Phi_{\Psi}(z) & =\Delta_{\Lambda}{ }^{-*}(z)\left(W_{\Psi}(z) W_{\Psi}^{*}(z)\right) \Delta_{\Lambda}{ }^{-1}(z) \\
& =\Delta_{\Lambda}{ }^{-*}(z)\left(H_{\Psi}^{*}(z) H_{\Psi}(z)\right) \Delta_{\Lambda}{ }^{-1}(z) \\
& =\left(H_{\Psi}(z) \Delta_{\Lambda}^{-1}(z)\right)^{*}\left(H_{\Psi}(z) \Delta_{\Lambda}{ }^{-1}(z)\right) \\
& =W_{1}(z) W_{1}^{*}(z)
\end{aligned}
$$

Finally, 44 can be computed obtaining a realization of $G(z) \Delta_{\Lambda}{ }^{-1}(z) W_{1}(z)$ and applying Lemma 6.1 Now, let $\Phi_{\Sigma}(z)=$ $\Delta_{\Lambda}{ }^{-*}(z) G^{*}(z) \Sigma G(z) \Delta_{\Lambda}{ }^{-1}(z)$, where $\Sigma$ is one of the precomputed generators of Range $\Gamma$. Then

$$
\begin{aligned}
& \int G Q_{\Lambda}^{-1}\left[\left(G^{*} \Sigma G Q_{\Lambda}^{-1} \Psi\right)+\left(G^{*} \Sigma G Q_{\Lambda}^{-1} \Psi\right)^{*}\right] Q_{\Lambda}^{-1} G^{*} \\
& =\int G \Delta_{\Lambda}^{-1} \Delta_{\Lambda}{ }^{*}\left[\left(G^{*} \Sigma G \Delta_{\Lambda}{ }^{-1} \Delta_{\Lambda}{ }^{-*} \Psi\right)\right. \\
& \left.+\left(\Psi \Delta_{\Lambda}{ }^{-1} \Delta_{\Lambda}{ }^{-*} G^{*} \Sigma G\right)\right] \Delta_{\Lambda}{ }^{-1} \Delta_{\Lambda}{ }^{-*} G^{*} \\
& =\int G \Delta_{\Lambda}{ }^{-1}\left[\left(\Delta_{\Lambda}{ }^{-*} G^{*} \Sigma G \Delta_{\Lambda}{ }^{-1} \Delta_{\Lambda}{ }^{-*} \Psi \Delta_{\Lambda}{ }^{-1}\right)\right. \\
& \left.+\left(\Delta_{\Lambda}{ }^{-*} \Psi \Delta_{\Lambda}{ }^{-1} \Delta_{\Lambda}{ }^{-*} G^{*} \Sigma G \Delta_{\Lambda}{ }^{-1}\right)\right] \Delta_{\Lambda}{ }^{-*} G^{*} \\
& =\int G \Delta_{\Lambda}^{-1}\left[\Phi_{\Sigma} \Phi_{\Psi}+\Phi_{\Psi} \Phi_{\Sigma}\right] \Delta_{\Lambda}{ }^{-*} G^{*} \\
& =\int G \Delta_{\Lambda}^{-1}\left[\left(\Phi_{\Sigma}+\Phi_{\Psi}\right)\left(\Phi_{\Sigma}+\Phi_{\Psi}\right)\right. \\
& \left.-\Phi_{\Psi} \Phi_{\Psi}-\Phi_{\Sigma} \Phi_{\Sigma}\right] \Delta_{\Lambda}{ }^{-*} G^{*} \\
& =\int G \Delta_{\Lambda}{ }^{-1}\left[\left(\Phi_{\Sigma}+\Phi_{\Psi}\right)\left(\Phi_{\Sigma}+\Phi_{\Psi}\right)\right] \Delta_{\Lambda}{ }^{-*} G^{*} \\
& -\int G \Delta_{\Lambda}{ }^{-1}\left[\Phi_{\Psi} \Phi_{\Psi}\right] \Delta_{\Lambda}{ }^{-*} G^{*}-\int G \Delta_{\Lambda}{ }^{-1}\left[\Phi_{\Sigma} \Phi_{\Sigma}\right] \Delta_{\Lambda}{ }^{-*} G^{*}
\end{aligned}
$$

which is a difference of integrals of the form (43). To compute [46, we must obtain (left) spectral factors of $\Phi_{\Psi} \Phi_{\Psi}{ }^{*}, \Phi_{\Sigma} \Phi_{\Sigma}^{*}$ and $\left(\Phi_{\Sigma}+\Phi_{\Psi}\right)\left(\Phi_{\Sigma}+\Phi_{\Psi}\right)^{*}$. Suppose, first, that $\Sigma>0$. For the first spectrum we have

$$
\begin{aligned}
\Phi_{\Psi} \Phi_{\Psi}{ }^{*} & =W_{1}\left(W_{1}^{*} W_{1}\right) W_{1}^{*}=W_{1}\left(H_{1} H_{1}^{*}\right) W_{1}^{*} \\
& =\left(W_{1} H_{1}\right)\left(W_{1} H_{1}\right)^{*}
\end{aligned}
$$

For the second, we have

$$
\begin{aligned}
\Phi_{\Sigma} & =\left(\Delta_{\Lambda}{ }^{-*} G^{*} \Sigma^{1 / 2}\right)\left(\Sigma^{1 / 2} G \Delta_{\Lambda}{ }^{-1}\right)=H_{\Sigma}^{*} H_{\Sigma}=W_{\Sigma} W_{\Sigma}^{*} \\
\Phi_{\Sigma} \Phi_{\Sigma}^{*} & =W_{\Sigma}\left(W_{\Sigma}^{*} W_{\Sigma}\right) W_{\Sigma}^{*}=W_{\Sigma}\left(K_{\Sigma} K_{\Sigma}^{*}\right) W_{\Sigma}^{*} \\
& =\left(W_{\Sigma} K_{\Sigma}\right)\left(W_{\Sigma} K_{\Sigma}\right)^{*}
\end{aligned}
$$


And for the third:

$$
\begin{aligned}
& \left(\Phi_{\Sigma}+\Phi_{\Psi}\right)\left(\Phi_{\Sigma}+\Phi_{\Psi}\right)^{*} \\
& =\left(Z_{\Sigma}+Z_{\Sigma}^{*}+Z_{1}+Z_{1}^{*}\right)\left(Z_{\Sigma}+Z_{\Sigma}^{*}+Z_{1}+Z_{1}^{*}\right)^{*} \\
& =\left(\left(Z_{\Sigma}+Z_{1}\right)+\left(Z_{\Sigma}+Z_{1}\right)^{*}\right)\left(\left(Z_{\Sigma}+Z_{1}\right)+\left(Z_{\Sigma}+Z_{1}\right)^{*}\right)^{*} \\
& =\left(Z_{1 \Sigma}+Z_{1 \Sigma}^{*}\right)\left(Z_{1 \Sigma}+Z_{1 \Sigma}^{*}\right)^{*} \\
& =W_{1 \Sigma}\left(W_{1 \Sigma}^{*} W_{1 \Sigma}\right) W_{1 \Sigma}^{*} \\
& =W_{1 \Sigma}\left(H_{1 \Sigma} H_{1 \Sigma}^{*}\right) W_{1 \Sigma}^{*} \\
& =\left(W_{1 \Sigma} H_{1 \Sigma}\right)\left(W_{1 \Sigma} H_{1 \Sigma}\right)^{*}
\end{aligned}
$$

where $Z_{1}$ is the causal part of $\Phi_{\Psi}, Z_{1 \Sigma}=Z_{1}+Z_{\Sigma}, W_{1 \Sigma}$ is a left factor of the spectrum $Z_{1 \Sigma}+Z_{1 \Sigma}^{*}, H_{1 \Sigma}$ is a left factor of the spectrum $W_{1 \Sigma}^{*} W_{1 \Sigma}$, and where we used Lemma 6.1 to obtain the causal part of $\Phi_{\Sigma}$ and $\Phi_{\Psi}$ from their spectral factors, and Lemma 6.2 to obtain the minimum phase spectral factor of the sum $\Phi_{\Sigma}+\Phi_{\Psi}$ from its causal part. Thus, if $\Sigma>0$, we really have all the tools to compute integral (46).

Now, $\Sigma$ is not necessarily positive definite, but if $-\lambda<0$ is the minimum between the eigenvalues of all the generators $\Sigma_{k}$, then $\Sigma+(\lambda+1) I$ is positive definite. Thus, in the general case, by linearity 46 can be reduced to:

$$
\begin{aligned}
& \int G \Delta_{\Lambda}{ }^{-1}\left[\Phi_{\Sigma} \Phi_{\Psi}+\Phi_{\Psi} \Phi_{\Sigma}\right] \Delta_{\Lambda}{ }^{-*} G^{*} \\
& =\int G \Delta_{\Lambda}{ }^{-1}\left[\Phi_{\Sigma+(\lambda+1) I-(\lambda+1) I} \Phi_{\Psi}\right. \\
& \left.\quad+\Phi_{\Psi} \Phi_{\Sigma+(\lambda+1) I-(\lambda+1) I}\right] \Delta_{\Lambda}{ }^{-*} G^{*} \\
& =\int G \Delta_{\Lambda}{ }^{-1}\left[\Phi_{\Sigma+(\lambda+1) I} \Phi_{\Psi}+\Phi_{\Psi} \Phi_{\Sigma+(\lambda+1) I}\right] \Delta_{\Lambda}{ }^{-*} G^{*} \\
& -(\lambda+1) \int G \Delta_{\Lambda}{ }^{-1}\left[\Phi_{I} \Phi_{\Psi}+\Phi_{\Psi} \Phi_{I}\right] \Delta_{\Lambda}{ }^{-*} G^{*}
\end{aligned}
$$

which is a difference of integrals with the same structure of (46), and that are computable with the above tools (obviously $\int G \Delta_{\Lambda}{ }^{-1}\left[\Phi_{I} \Phi_{\Psi}+\Phi_{\Psi} \Phi_{I}\right] \Delta_{\Lambda}{ }^{* *} G^{*}$ needs to be computed only once). This enables us to solve equation 24 .

\section{Computations in the backtracking step}

The backtracking stage involves similar, though easier, computations. We must check the following conditions:

$$
\begin{gathered}
\Lambda_{i}+t_{i}^{k} \Delta \Lambda_{i} \in \mathcal{L}_{\Gamma}^{H} \\
J_{\Psi}\left(\Lambda_{i}+t_{i}^{k} \Delta \Lambda_{i}\right)<J_{\Psi}\left(\Lambda_{i}\right)+\alpha t_{i}^{k} \nabla J_{\Psi \Lambda_{i}} \Delta \Lambda_{i}
\end{gathered}
$$

Checking 51 is really a matter of checking whether we can factorize $I+G^{*}\left(\Lambda_{i}+t_{i}^{k} \Delta \Lambda_{i}\right) G$. Thus $t_{i}^{k}$ must be halved until the ARE 40 is solvable having $\Lambda=\Lambda_{i}+t_{i}^{k} \Delta \Lambda_{i}$.

Finally, to check (52), we need to compute $J_{\Psi}$. This can be done in a way similar to the above computations:

$$
\begin{aligned}
J_{\Psi}(\Lambda) & =\operatorname{tr} \int\left(I+G^{*} \Lambda G\right)^{-1} \Psi+\operatorname{tr} \Lambda \\
& =\operatorname{tr} \int \Delta_{\Lambda}{ }^{-1} \Delta_{\Lambda}{ }^{-*} W_{\Psi} W_{\Psi}^{*}+\operatorname{tr} \Lambda \\
& =\operatorname{tr} \int \Delta_{\Lambda}{ }^{-*}\left(W_{\Psi} W_{\Psi}^{*}\right) \Delta_{\Lambda}{ }^{-1}+\operatorname{tr} \Lambda \\
& =\operatorname{tr} \int \Delta_{\Lambda}{ }^{-*}\left(H_{\Psi}^{*} H_{\Psi}\right) \Delta_{\Lambda}{ }^{-1}+\operatorname{tr} \Lambda \\
& =\operatorname{tr} \int\left(H_{\Psi} \Delta_{\Lambda}{ }^{-1}\right)^{*}\left(H_{\Psi} \Delta_{\Lambda}{ }^{-1}\right)+\operatorname{tr} \Lambda \\
& =\operatorname{tr} \int W W^{*}+\operatorname{tr} \Lambda
\end{aligned}
$$

\section{PRoof of GLOBAL CONVERGENCE}

Given that the minimum of $J_{\Psi}$ exists and is unique, we investigate global convergence of our Newton algorithm. First, we recall the following

Definition: a function $f(x)$ twice differentiable in a set $S$ is said to be strongly convex in $S$ if there exists a constant $m>0$ such that $H(x) \geq m I$ for $x \in S$, where $H(x)$ is the Hessian of $f$ at $x$. 
We restrict our analysis to a sublevel set of $J_{\Psi}$. Let $\Lambda_{0}=0$. The set

$$
S:=\left\{\Lambda \in \mathcal{L}_{\Gamma}^{H}: J_{\Psi}(\Lambda) \leq J_{\Psi}\left(\Lambda_{0}\right)=\operatorname{tr} \int \Psi\right\}
$$

is compact (as it was shown in [17, Section VII]). Because of the backtracking in the algorithm, the sequence $J_{\Psi}\left(\Lambda_{0}\right), J_{\Psi}\left(\Lambda_{1}\right), \ldots$ is decreasing. Thus $\Lambda_{n} \in S, \forall n \geq 0$. We now wish to apply a theorem in [10, 9.5.3, p. 488] on convergence of the Newton algorithm with backtraking for strongly convex functions on $\mathbb{R}^{n}$. This theorem ensures linear decrease for a finite number of steps, and quadratic convergence to the minimum after the linear stage, thus establishing global convergence of the Newton algorithm with backtracking. We proceed to establish first strong convexity of $J_{\Psi}$ on $S$. To do that, we employ the following result.

Lemma 7.1: Let $f(x)$ be defined over an open convex subset $D$ of a finite-dimensional linear space $V$. Assume that $f$ is twice continuously differentiable and strictly convex on $D$. Then $f$ is strongly convex on any compact set $S \subset D$.

Proof:

First, recall that since $f$ is twice continuously differentiable and strictly convex, its Hessian $H_{x}$ is an Hermitian positivedefinite matrix at each point $x$. By Lemma 5.1, the mapping from $H$ to its minimum (real) eigenvalue is continuous. It follows that the mapping from $x$ to the minimum eigenvalue of the Hessian of $f$ at $x$ is also continuous, being a composition of continuous functions. Hence the latter admits a minimum $m$ in the compact set $S$ by Weierstrass' theorem. Thus $m$ is the minimum of the eigenvalues of all the Hessians computed in $S$, and $m$ cannot be zero, since otherwise there would be an $x$ with $H_{x}$ singular, and this cannot happen since $f$ is strictly convex. Hence $H_{x} \geq m I \forall x \in S$, i.e. $f$ is strongly convex on $S$.

Remark 7.2: By an argument similar to that of Lemma 5.1, it can be shown that for a twice continuously differentiable function which is strictly convex on $D$, there exists $M>0$ such that $H_{x} \leq M I$ for all $x \in S$. Moreover, strong convexity on a closed set $S$ implies boundedness of the latter. Thus, strong convexity and boundedness of the Hessian are intertwined, and both are essential in the proof of Theorem 7.3 (see [10]).

Theorem 7.3: The following facts hold true:

1) $J_{\Psi}$ is twice continuously differentiable on $S$;

2) $J_{\Psi}$ is strongly convex on $S$;

3) the Hessian of $J_{\Psi}$ is Lipschitz-continuous over $S$;

4) the sequence $\left\{\Lambda_{i} ; i \geq 0\right\}$ generated by the Newton algorithm of Section $V(23)-52$ converges to the unique minimum point of $J_{\Psi}$ in $\mathcal{L}_{\Gamma}^{H}$.

Proof:

Property 1 is a trivial consequence of Theorem 5.3. To prove 2 , remember that $J_{\Psi}$ is strictly convex on $\mathcal{L}_{\Gamma}^{H}$, hence also on $S$, and apply Lemma 7.1. As for property 3, what it really says is that the following operator:

$$
\mathcal{H}: \Lambda \mapsto H_{\Lambda}(\cdot, \cdot)
$$

is Lipschitz continuous on $S$. Theorem 5.3 implies that $J_{\Psi} \in C^{3}\left(\mathcal{L}_{\Gamma}^{H}\right)$ or, which is the same, that $\mathcal{H} \in C^{1}\left(\mathcal{L}_{\Gamma}^{H}\right)$. The continuous differentiability of $\mathcal{H}$ implies its Lipschitz continuity over an arbitrary compact subset of $\mathcal{L}_{\Gamma}^{H}$, hence also over the sublevel set $S$, and property 3 follows. Finally, to prove 4, notice that all the hypotheses of [10, 9.5.3, p. 488] are satisfied. Namely, the function to be minimized $J_{\Psi}$ is strongly convex on the compact set $S$, and its Hessian is Lipschitz-continuous over $S$. It remains to observe that $J_{\Psi}$ is defined over a subset of the linear space Range $\Gamma$ which has finite dimension $d$ over $\mathbb{R}$ (recall that Range $\Gamma$ is spanned by a finite set of matrices. See Proposition 3.1 and Remark 3.3, where $d \leq N$ ). Thus, once we choose a base in Range $\Gamma$, to every $\Lambda \in \mathcal{L}_{\Gamma}^{H}$ there corresponds a vector in $\mathbb{R}^{d}$, to every positive definite bilinear form over Range $\Gamma$ there corresponds a positive definite matrix in $\mathbb{R}^{d \times d}$, and to every compact set in $\mathcal{L}_{\Gamma}^{H}$ there corresponds a compact set in $\mathbb{R}^{d}$. Hence, every convergence result that holds in $\mathbb{R}^{d}$ must also hold in the abstract setting, in view of the homeomorphism between one space and the other.

\section{ApPLiCATION TO SPECTRUM ESTIMATION}

\section{A. A spectral estimation procedure}

Following the purposes of the THREE method presented in [9], now we describe an application of the above approximation algorithm to the estimation of spectral densities. Consider first the scalar case, and suppose that the finite sequence $y_{1}, \ldots, y_{N}$ is extracted from a realization of a zero-mean, weakly stationary discrete-time process $\left\{y_{t}\right\}_{t=-\infty}^{+\infty}$. We want to estimate the spectral density $\Phi_{y}\left(\mathrm{e}^{\mathrm{j} \vartheta}\right)$ of $y$. The idea is the following:

- Fix a transfer function $G(z)=(z I-A)^{-1} B$, feed the data $\left\{y_{i}\right\}$ to it, and collect the output data $\left\{x_{i}\right\}$.

- Compute a consistent, and possibly unbiased, estimate $\hat{\Sigma}$ of the covariance matrix of the outputs $\left\{x_{i}\right\}$. Note that some output samples $x_{1}, \ldots, x_{M}$ should be discarded so that the filter can be considered to operate in steady state.

- Choose as "prior" spectrum $\hat{\Phi}_{y}$ a coarse, low-order, estimate of the true spectrum of $y$ obtained by means of another (simple) identification method. 
- "Refine" the estimate $\hat{\Phi}_{y}$ by solving the approximation problem 7 with respect to $G(z), \Sigma=\hat{\Sigma}$, and $\Psi=\hat{\Phi}_{y}$.

To be clear, the result of the above procedure is the only spectrum, compatible with the output variance $\hat{\Sigma}$, which is closest to the rough estimate $\hat{\Phi}_{y}$ in the $d_{H}$ distance. Note that we are left with significant degrees of freedom in applying the above procedure: The method for estimating $\hat{\Phi}_{y}$, in particular its degree, and the whole structure of $G(z)=(z I-A)^{-1} B$, which has no contraints other than $A$ being a stability matrix and $(A, B)$ being reachable.

The coarsest possible estimate of $\Phi_{y}$ is the constant spectrum equal to the sample variance of the $\left\{y_{i}\right\}$, i.e. $\hat{\Phi}_{y}\left(\mathrm{e}^{\mathrm{j} \vartheta}\right) \equiv$ $\hat{\sigma}_{y}^{2}$, where $\hat{\sigma}_{y}^{2}=\frac{1}{N-1} \sum_{i=1}^{N}\left|y_{i}\right|^{2}$. The resulting spectrum has the form $\hat{\sigma}_{y}^{2}\left(1+G^{*} \hat{\Lambda} G\right)^{-2}$. Another simple choice is $\hat{\Phi}_{y}=$ $W(z) W^{*}(z)$, where $W(z)=\hat{\sigma}_{e} \frac{c(z)}{a(z)}$ is a low-order AR, MA or ARMA model estimated from $y_{1}, \ldots, y_{N}$ by means of predictive error minimization methods or the like.

The flexibility in the choice of $G(z)$ is more essential, and has more profound implications. As described in [9], [28], [15] and [17], the following choice:

$$
A=\left[\begin{array}{ccccc}
p_{1} & 0 & 0 & \ldots & 0 \\
0 & p_{2} & 0 & \ldots & 0 \\
\vdots & \vdots & & \ddots & \vdots \\
0 & 0 & 0 & \ldots & 0 \\
0 & 0 & 0 & \ldots & p_{n}
\end{array}\right], \quad B=\left[\begin{array}{c}
1 \\
1 \\
\vdots \\
1 \\
1
\end{array}\right]
$$

where the $p_{i}$ 's lie inside the unit circle, implies that the (true) steady-state variance $\Sigma$ has the structure of a Pick matrix, and the corresponding problem of finding any spectrum that satisfies (1) is a Nevanlinna-Pick interpolation. Moreover, the following choice:

$$
A=\left[\begin{array}{ccccc}
0 & 1 & 0 & \ldots & 0 \\
0 & 0 & 1 & \ldots & 0 \\
\vdots & \vdots & & \ddots & \vdots \\
0 & 0 & 0 & \ldots & 1 \\
0 & 0 & 0 & \ldots & 0
\end{array}\right], \quad B=\left[\begin{array}{c}
0 \\
0 \\
\vdots \\
0 \\
1
\end{array}\right]
$$

implies that the steady-state variance $\Sigma$ is a Toeplitz matrix whose diagonals contain the lags $c_{0}, c_{1}, \ldots, c_{n-1}$ of the covariance signal of the input, and the corresponding problem of finding any spectrum that satisfies (1) is a covariance extension problem.

These facts justify the theoretical interest in algorithms for constrained spectrum approximation, if for no other reason, as tools to compute at least one solution to a Nevanlinna-Pick interpolation or to a covariance extension problem, respectively. But the freedom in choosing $G(z)$ has implications also in the above practical application to spectral estimation, where the key properties, not surprisingly, depend on the poles of $G(z)$, i.e., the eigenvalues of $A$. In general, as described in [9], the magnitude of the latter has implications on the variance of the sample covariance $\hat{\Sigma}$ : The closer the eigenvalues to the origin, the smaller that variance (see [9. Section II.D]). Moreover, at least as far as THREE [9] is concerned, the phase of the eigenvalues influences resolution capability: More precisely, the spectrum estimation procedure has higher resolution in those sectors of the unit circle where more eigenvalues are located. According to simulations, the latter statement appears to be true also in our setting (the fundamental difference being that the metric which is minimized is the Hellinger distance instead of the Kullback-Leibler one).

Remark 8.1: In the above setting $\hat{\Sigma}$ is a consistent estimate of the true steady-state variance. Although $\hat{\Sigma}$ must belong to Range $\Gamma$ as $N \rightarrow+\infty$ (this being the case even if $y$ is the sum of a purely nondeterministic process and some sinusoids, as in the simulations that follow), it is almost certainly not the case that $\hat{\Sigma} \in \operatorname{Range} \Gamma$ when we have available only the finitely many data $x_{M+1}, \ldots, x_{N}$. Strictly speaking, this implies that the contraint 11 with $\Sigma=\hat{\Sigma}$ is almost always not feasible. It turns out that, increasing the tolerance threshold in its step 5, the Newton algorithm exhibits some kind of robustness in this respect. That is, it leads to a $\Lambda$ whose corresponding spectrum $\hat{\Phi}$ is close to satisfying the constraint.

Nevertheless, we prefer a clear understanding of what the resulting spectrum really is. Thus, we choose to enforce feasibility of the approximation problem, at least as permitted by machine number representation, before starting the optimization procedure. To this end, following the same approach employed in [9], we pose the approximation problem not in terms of the estimated $\hat{\Sigma}$, but in terms of its orthogonal projection $\hat{\Sigma}_{\Gamma}$ onto Range $\Gamma$, which can be easily computed by means of algebraic methods. That is to say: We cannot approximate in the preimage $\Gamma^{-1}(\hat{\Sigma})$, because that set is empty, thus we choose to approximate in $\Gamma^{-1}\left(\hat{\Sigma}_{\Gamma}\right)$, where $\hat{\Sigma}_{\Gamma}$ is the matrix closest to $\hat{\Sigma}$ such that its preimage is not empty. This seems a reasonable choice and by the way it is, mutatis mutandis, what the Moore-Penrose pseudoinverse does for the "solution" $\hat{x}=A^{\dagger} b$, when the linear system $A x=b$ is not solvable.

Note that it is not guaranteed at all that the projection of a positive definite matrix onto a subspace of the Hermitian matrices is itself positive definite. In practice, this is not really a problem, inasmuch $\hat{\Sigma}$ is "sufficiently positive" and close to Range $\Gamma$. The positivity of $\hat{\Sigma}_{\Gamma}$ must anyway be checked before proceeding. This approach and the considerations on the positivity issue should be compared to [9. Section II.D], which deals with the particular case when Range $\Gamma$ is the space of Toeplitz matrices, and to [27, Section 4], where, to find a matrix a $\hat{\Sigma}_{\Gamma}$ close to $\hat{\Sigma}$, a Kullback-Leibler criterion is adopted instead of least squares. 


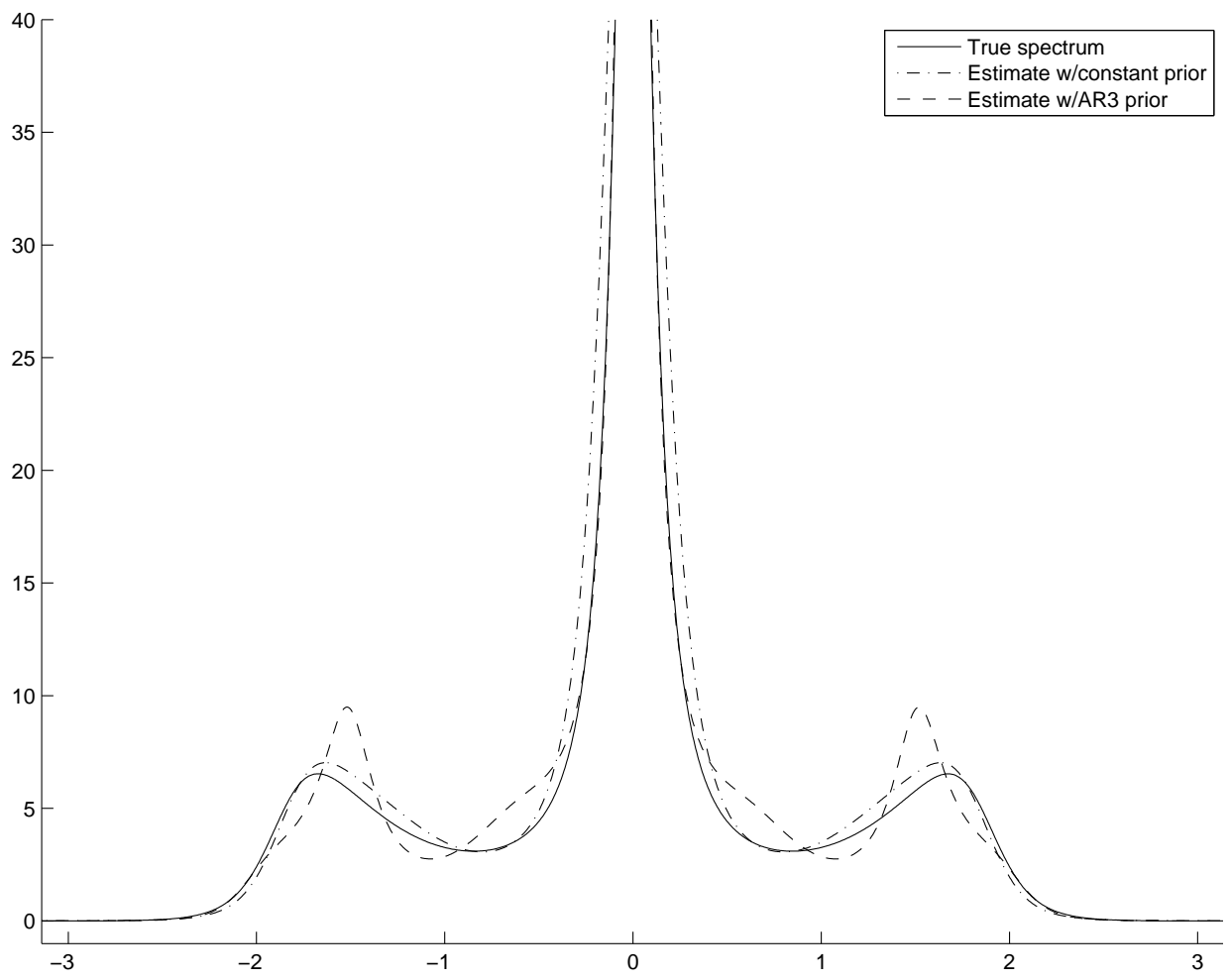

Fig. 1. Estimation of an $\operatorname{ARMA}(6,4)$ spectrum by means of Hellinger-distance spectrum approximation, constant prior and $\mathrm{AR}(3)$ prior.

Figure 1 shows the results of the above estimation procedure with $G(z)$ structured according to the covariance extension setting (56) with 6 covariance lags (i.e. $n=6, A$ is $6 \times 6$ ), run over 500 samples of the following ARMA process:

$$
\begin{aligned}
y(t) & =0.5 y(t-1)-0.42 y(t-2)+0.602 y(t-3) \\
& -0.0425 y(t-4)+0.1192 y(t-5) \\
& +e(t)+1.1 e(t-1)+0.08 e(t-2)-0.15 e(t-3)
\end{aligned}
$$

(poles in $0.9,-0.2 \pm 0.7 \mathrm{j}, \pm 0.5 \mathrm{j}$ ) where $e(t)$ is a zero-mean Gaussian white noise with unit variance. Two priors, both estimated from data, have been considered: the constant spectrum $\hat{\Phi}_{y}\left(\mathrm{e}^{\mathrm{j} \vartheta}\right) \equiv \hat{\sigma}_{y}^{2}$ and the spectrum $\hat{\Phi}_{y}=W_{A R}(z) W_{A R}^{*}(z)$, where $W_{A R}(z)=\frac{\hat{\sigma}_{e}}{a(z)}$ is an AR model of order 3 obtained from the data by means of the Predictive Error Method procedure in Matlab's System Identification toolbox.

Figure 2 shows the performance of the above procedure in a setting that resembles that of [9, Section IV.B, Example 1]. The estimation procedure was run on 300 samples of a superposition of two sinusoids in colored noise:

$$
\begin{aligned}
& y(t)=0.5 \sin \left(\omega_{1} t+\phi_{1}\right)+0.5 \sin \left(\omega_{2} t+\phi_{2}\right)+z(t) \\
& z(t)=0.8 z(t-1)+0.5 \nu(t)+0.25 \nu(t-1)
\end{aligned}
$$

with $\phi_{1}, \phi_{2}$ and $\nu(t)$ independent normal random variables with zero mean and unit variance, $\omega_{1}=0.42$ and $\omega_{2}=0.53$. The prior here considered is the constant spectrum equal to the sample variance of the $\left\{y_{i}\right\}$ data. Following [9], $A$ was chosen real block-diagonal with the following poles (equispaced in a narrow range where the frequencies of the two sinusoids lie, to increase resolution in that region):

$$
\begin{aligned}
& 0,0.85,-0.85 \\
& 0.9 \mathrm{e}^{ \pm \mathrm{j} 0.42}, 0.9 \mathrm{e}^{ \pm \mathrm{j} 0.44}, 0.9 \mathrm{e}^{ \pm \mathrm{j} 0.46}, 0.9 \mathrm{e}^{ \pm \mathrm{j} 0.48}, 0.9 \mathrm{e}^{ \pm \mathrm{j} 0.50}
\end{aligned}
$$

(and $B$ a column of ones). It can be seen that Hellinger-distance based approximation does a good job, as does the THREE algorithm, at detecting the spectral lines at frequencies $\omega_{1}$ and $\omega_{2}$. 


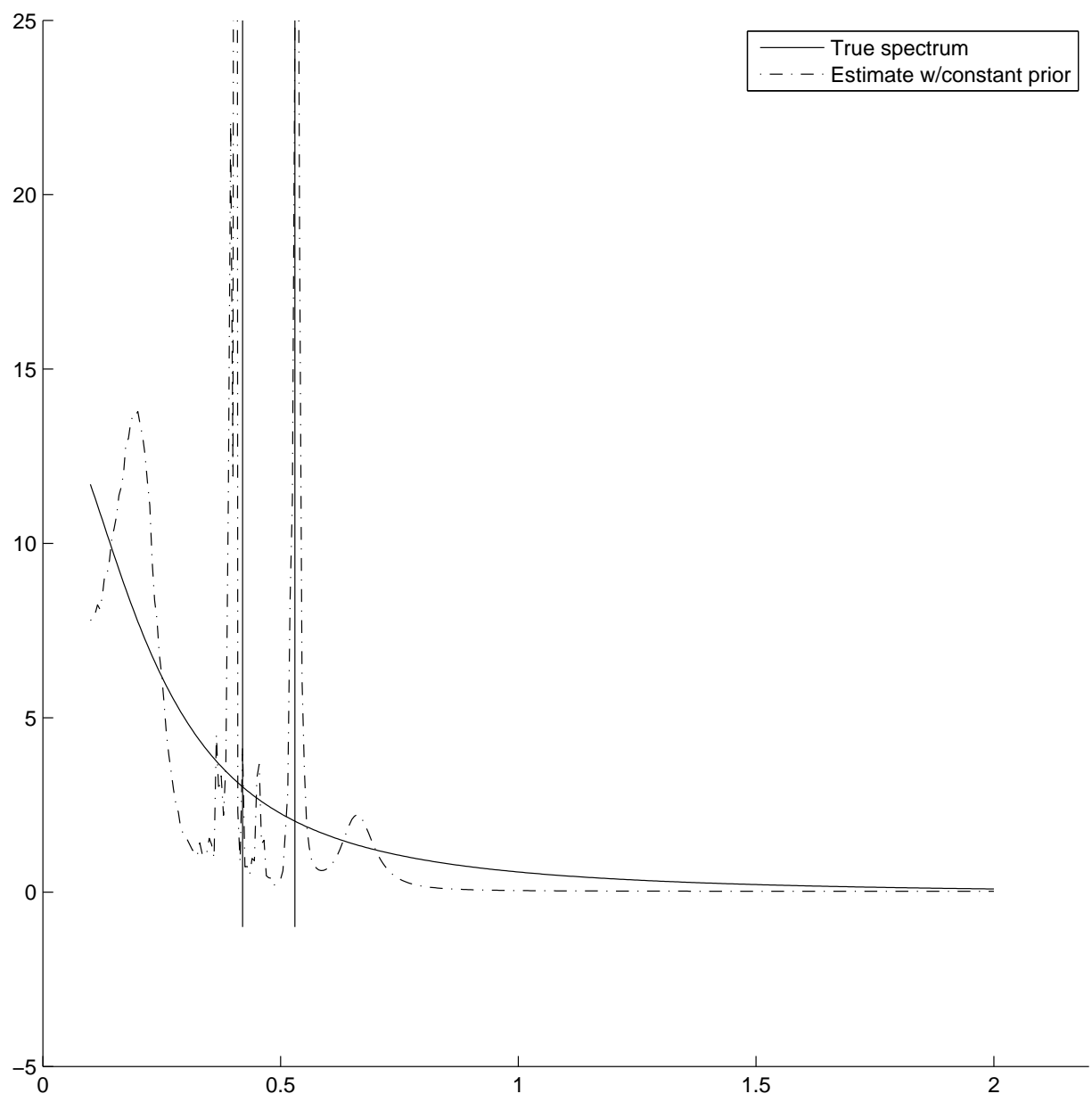

Fig. 2. Spectral estimates of two sinusoids with superimposed noise by means of Hellinger-distance spectrum approximation, constant prior. Compare with [9. Section IV.B, Example 1].

\section{Simulation results: Multivariate case}

We now consider spectral estimation for a multivariate process. Here, 100 samples of a bivariate process with a high order spectrum were generated by feeding a bivariate Gaussian white noise with mean 0 and variance $I$ to a square (stable) shaping filter of order 40. The latter was constructed with random coefficients, except for one fixed conjugate pair of poles with radius 0.9 and argument 0.52 , and one fixed conjugate pair of zeros with radius $1-10^{-5}$ and argument 0.2 . The transfer function $G(z)$ was chosen with one pole in the origin and 4 complex pole pairs with radius 0.9 and frequencies equispaced in the range $[0, \pi]$. Then the above estimating procedure was applied, with prior spectrum chosen as the constant density equal to the sample covariance of the bivariate process $y$. Figure 3 shows a plot of $\Phi_{11}\left(\mathrm{e}^{\mathrm{j} \vartheta}\right), \operatorname{Re} \Phi_{12}\left(\mathrm{e}^{\mathrm{j} \vartheta}\right), \operatorname{Im} \Phi_{12}\left(\mathrm{e}^{\mathrm{j} \vartheta}\right)$ and $\Phi_{22}\left(\mathrm{e}^{\mathrm{j} \vartheta}\right)$, respectively for the true spectrum and for the estimation of the latter based on one run of 100 samples. In Figure 4 we compare the performances of various spectral estimation methods in the following way. We consider four estimates $\Phi_{\mathrm{H}}, \hat{\Phi}_{\mathrm{ME}}, \hat{\Phi}_{\mathrm{PEM}}$, and $\hat{\Phi}_{\mathrm{N} 4 \mathrm{SID}}$ of $\Phi$. The spectral density $\hat{\Phi}_{\mathrm{H}}$ is the estimate obtained by the procedure described above in Subsection VIII-A The spectral density $\hat{\Phi}_{\mathrm{ME}}$ is the maximum entropy estimate [22] obtained using the same $G(z)$ employed to obtain our estimate. The spectral densities $\hat{\Phi}_{\mathrm{PEM}}$ and $\hat{\Phi}_{\mathrm{N} 4 \mathrm{SID}}$ are the estimates of $\Phi$ obtained by using "off-the-shelf" Matlab procedures for the Prediction Error Method (see i.e. [39] or [33]) and for the N4SID method (see [42] or [33]): The former is a multivariable extension of the classical approach to ARMAX identification, while the latter is a standard algorithm in the modern field of subspace identification. In order to obtain a comparison reasonably independent of the specific data set, we have performed 50 independent runs each with 100 samples of $y$. In such a way we have obtained 50 different estimates $\hat{\Phi}_{\mathrm{M}, i}, \mathrm{M}=\mathrm{H}, \mathrm{ME}, \mathrm{PEM}, \mathrm{N} 4 \mathrm{SID}, i=1,2, \ldots, 50$, for each method.

We have then defined

$$
E_{\mathrm{H}}(\vartheta):=\frac{1}{50} \sum_{i=1}^{50}\left\|\hat{\Phi}_{\mathrm{H}, i}\left(\mathrm{e}^{j \vartheta}\right)-\Phi\left(\mathrm{e}^{j \vartheta}\right)\right\|,
$$

where $\|\cdot\|$ denotes the spectral norm. This is understood as the average estimation error of our method at each frequency. 

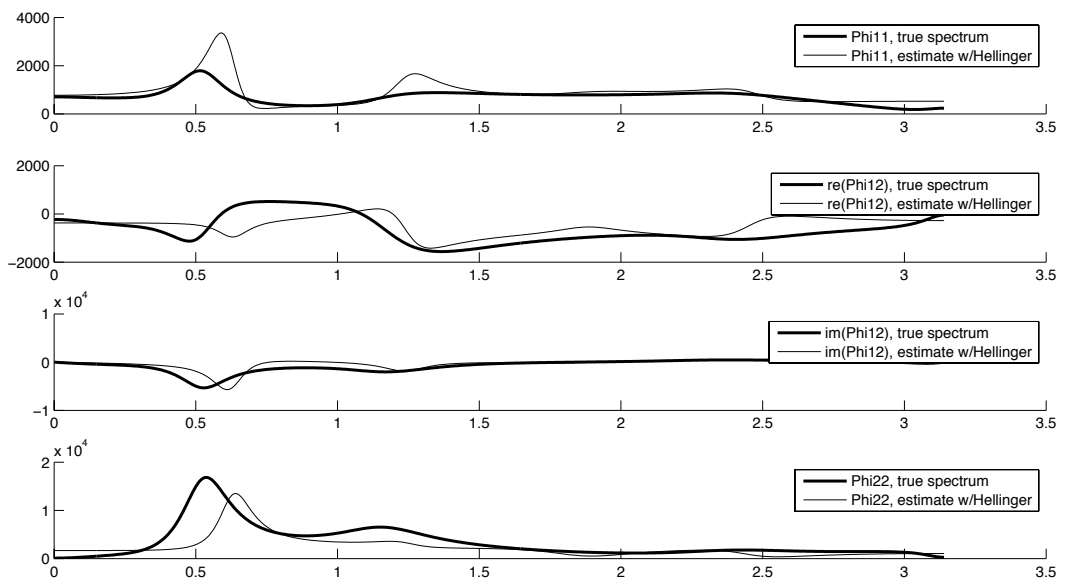

Fig. 3. Estimation of the spectrum of a bivariate process with rich dynamics by means of Hellinger-distance spectrum approximation, constant prior.
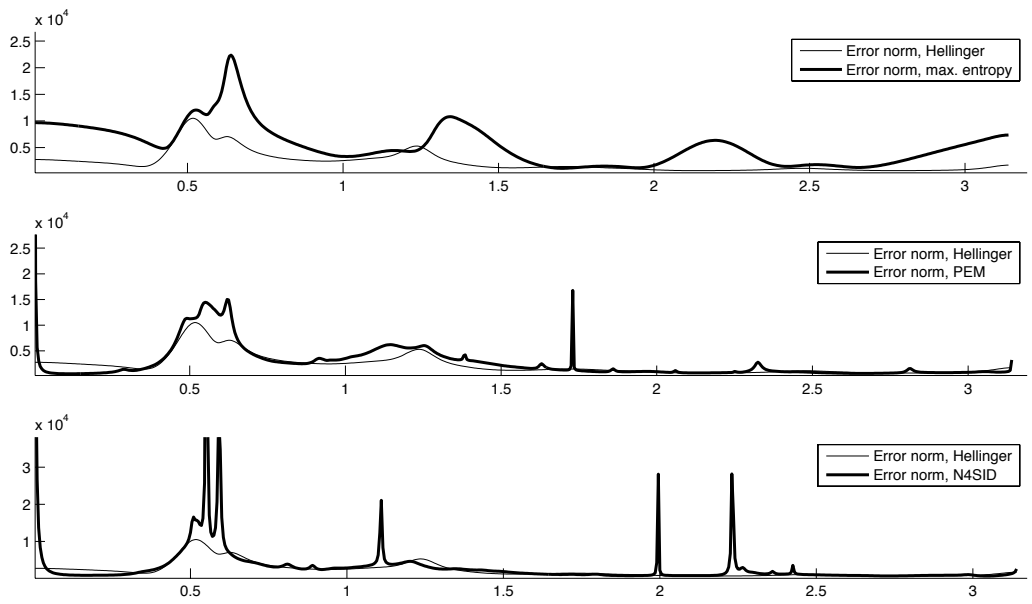

Fig. 4. Estimation of the spectrum of a bivariate process with rich dynamics by means of various methods. Comparison between the spectral norm of the differences $\hat{\Phi}_{\mathrm{H}}-\Phi, \hat{\Phi}_{\mathrm{ME}}-\Phi, \hat{\Phi}_{\mathrm{PEM}}-\Phi$, and $\hat{\Phi}_{\mathrm{N} 4 \mathrm{SID}}-\Phi$ (average over 50 simulations).

Similarly, we have defined the average errors $E_{\mathrm{ME}}(\vartheta), E_{\mathrm{PEM}}(\vartheta)$, and $E_{\mathrm{N} 4 \mathrm{SID}}(\vartheta)$ of the other methods. In the each of the plots of Figure 4, we depict the average error of our method $E_{\mathrm{H}}(\vartheta)$ together with the average error of one of the other methods. More explicitly, the first diagram shows the error for the Hellinger approximation method and for the maximum entropy spectrum described in [22]. The second diagram shows the error for the Hellinger approximation and for the spectrum obtained via MATLAB's PEM identification method. The third diagram shows the same for Hellinger approximation and MATLAB's N4SID method. The Hellinger approximation based approach appears to perform better or much better than the other methods. The simulation yields similar results with $N=200$ data points. With $N=300$ data samples, PEM and N4SID perform as well as our method.

Of course, one should always take into account the complexity of the resulting spectrum. In this example, $G(z)$ being of order 9 , the resulting spectral factor (or "model") produced by the Hellinger approximation has order 18, whereas the corresponding maximum entropy model has order 9 and both N4SID and PEM usually choose order 10.

In our simulation, the norm of the difference of two estimates produced by PEM or by N4SID is sometimes very large when compared to the norm of the difference between any two of the estimates produced by our method. That is, although PEM and N4SID are provably consistent as $N \rightarrow \infty$, when few data are available both of them may introduce occasional artifacts, which are well visible as "peaks" in figure 4 (a "peak" in the 50-run average is due to a very high error in one of the runs, not to a systematic error). Our method appears to be more robust in this respect. 


\section{CONCLUSION}

In this paper, we considered the the new approach to multivariate spectrum approximation problem with respect to the multivariable Hellinger distance, which was proposed in [17]. We developed in detail the matricial Newton algorithm which was sketched there, and proved its global convergence. Finally, we described an application of this approach to spectral estimation, and tested it against the well-known PEM and N4SID algorithms.

It appears that approximation in the Hellinger distance may be a useful tool to gain insight into the dynamics of a multivariate process when fewer data are available. In particular, simulations suggest that this method is less prone to produce artifacts than PEM and N4SID. Another advantage of our method and of the maximum entropy paradigm is that a higher resolution estimate in a prescribed frequency band can be easily achieved by properly placing some poles of $G(z)$ close to the unit circle and with phase in the prescribed band.

Numerical robustness of the algorithm with respect to the number and the position of the poles is an open challenge. Also, the analysis of the achievable precision of the results (in a statistical sense) has still to be developed.

\section{ACKNOWLEDGMENTS}

The detailed comments of the anonymous reviewers are gratefully acknowledged.

\section{REFERENCES}

[1] A.Blomqvist, A.Lindquist and R.Nagamune, Matrix-valued Nevanlinna-Pick interpolation with complexity constraint: An optimization approach, IEEE Trans. Aut. Control vol. 48, pp. 2172-2190, 2003.

[2] C. I. Byrnes, P. Enqvist and A. Linquist, Cepstral coefficients, covariance lags and pole-zero models for finite data strings, IEEE Trans. Sig. Proc. vol. 50, pp. 677-693, 2001.

[3] C. I. Byrnes, P. Enqvist and A. Linquist, Identifiability and well-posedness of shaping-filter parameterizations: A global analysis approach , SIAM J. Control and Optimization 41 (2002), 23-59.

[4] C. I. Byrnes, T. Georgiou, and A. Lindquist, A generalized entropy criterion for Nevanlinna-Pick interpolation with degree constraint, IEEE Trans. Aut. Control vol. 46, pp. 822-839, 2001.

[5] C. I. Byrnes, T. Georgiou, A. Lindquist and A. Megretski, Generalized interpolation in H-infinity with a complexity constraint, Trans. American Math. Society vol. 358(3), pp. 965-987, 2006 (electronically published on December 9, 2004).

[6] C. I. Byrnes, S. Gusev, and A. Lindquist, A convex optimization approach to the rational covariance extension problem, SIAM J. Control and Opimization vol. 37, pp. 211-229, 1999.

[7] C. I. Byrnes, S. Gusev, and A. Lindquist, From finite covariance windows to modeling filters: A convex optimization approach, SIAM Review vol. 43, pp. 645-675, 2001.

[8] C. I. Byrnes and A. Lindquist, The generalized moment problem with complexity constraint, Integral Equations and Operator Theory vol. 56(2), pp. 163-180, 2006.

[9] C. I. Byrnes, T. Georgiou, and A. Lindquist, A new approach to spectral estimation: A tunable high-resolution spectral estimator, IEEE Trans. Sig. Proc. vol. 49, pp. 3189-3205, 2000.

[10] S. Boyd, L. Vandenberghe, Convex Optimization, Cambridge University Press, Cambridge, UK, 2004.

[11] M. Deistler, A Birds Eye View on System Identification, in Modeling, Estimation and Control: Festschrift in honor of Giorgio Picci on the occasion of his sixty-fifth Birthday, A. Chiuso, A. Ferrante and S. Pinzoni (eds), Springer-Verlag, 2007.

[12] P. Colaneri, A. Ferrante, Algebraic Riccati equation and J-spectral factorization for $\mathcal{H}_{\infty}$ estimation, Systems \& control letters vol. 51, pp. 383-393, 2004.

[13] P. Enquist, A homotopy approach to rational covariance extension with degree constraint, Int. J. Appl. Math. and Comp. Sci. vol. 11, pp. 1173-1201, 2001.

[14] P. Faurre, M. Clerget, and F. Germain, Operateurs Rationnels Positifs, Dunod, Paris, 1979.

[15] A. Ferrante, M. Pavon and F. Ramponi, Constrained approximation in the Hellinger distance, Proceedings of the European Control Conference 2007 (ECC'07) pp. 322-327, Kos, Greece, July 2007.

[16] A. Ferrante, M. Pavon and F. Ramponi, Further results on the Byrnes-Georgiou-Lindquist generalized moment problem, in Modeling, Estimation and Control: Festschrift in honor of Giorgio Picci on the occasion of his sixty-fifth Birthday, A. Chiuso, A. Ferrante and S. Pinzoni (eds), Springer-Verlag, pp. 73-83, 2007.

[17] A. Ferrante, M. Pavon and F. Ramponi, Hellinger vs. Kullback-Leibler multivariable spectrum approximation, IEEE Trans. Aut. Control vol. 53, pp. 954-967, 2008

[18] T. Georgiou, The structure of state covariances and its relation to the power spectrum of the input, IEEE Trans. Aut. Control vol. 47, pp. 1056-1066, 2002.

[19] T. Georgiou, Realization of power spectra from partial covariance sequences, IEEE Trans. on Acoustics, Speech, and Signal Processing vol. 35, pp. 438-449, 1987.

[20] T. Georgiou, The interpolation problem with a degree constraint, IEEE Trans. Aut. Control vol. 44, pp. 631-635, 1999.

[21] T. Georgiou, Spectral estimation by selective harmonic amplification, IEEE Trans. Aut. Control vol. 46, pp. 29-42, 2001.

[22] T. Georgiou, Spectral analysis based on the state covariance: the maximum entropy spectrum and linear fractional parameterization, IEEE Trans. Aut. Control vol. 47, pp. 1811-1823, 2002.

[23] T. Georgiou, Solution of the general moment problem via a one-parameter imbedding, IEEE Trans. Aut. Control vol. 50, pp. 811-826, 2005.

[24] T. Georgiou, Relative entropy and the multivariable multidimensional moment problem, IEEE Trans. Inform. Theory vol. 52, pp. 1052-1066, 2006.

[25] T. Georgiou, Distances between power spectral densities, arXiv e-print math. OC/0607026.

[26] T. Georgiou, An intrinsic metric for power spectral density functions, arXiv e-print math. OC/0608486.

[27] T. Georgiou, Distances Between Time-Series and Their Autocorrelation Statistics, in Modeling, Estimation and Control: Festschrift in honor of Giorgio Picci on the occasion of his sixty-fifth Birthday, A. Chiuso, A. Ferrante and S. Pinzoni (eds), Springer-Verlag, pp. 123-133, 2007.

[28] T. Georgiou and A. Lindquist, Kullback-Leibler approximation of spectral density functions, IEEE Trans. Inform. Theory vol. 49, pp. 2910-2917, 2003.

[29] T. Georgiou and A. Lindquist, Remarks on control design with degree constraint, IEEE Trans. Aut. Control vol. AC-51, pp. 1150-1156, 2006.

[30] T. Georgiou and A. Lindquist, A convex optimization approach to ARMA modeling, IEEE Trans. Aut. Control, in press.

[31] P. Kosmol, Optimierung und Approximation, de Gruyter, Berlin, 1991.

[32] A. Lindquist, Prediction-error approximation by convex optimization, in Modeling, Estimation and Control: Festschrift in honor of Giorgio Picci on the occasion of his sixty-fifth Birthday, A. Chiuso, A. Ferrante and S. Pinzoni (eds), Springer-Verlag, pp. 265-275, 2007. 
[33] L. Ljung, System Identification: Theory for the User, Prentice Hall, Englewood Cliffs, 1987.

[34] M. Marden, Geometry of polynomials, American Mathematical Society, Providence, RI, 1966.

[35] R. Nagamune, A robust solver using a continuation method for Nevanlinna-Pick interpolation with degree constraint, IEEE Trans. Aut. Contr., vol 48, pp. 113-117, 2003

[36] A. Nasiri Amini, E. Ebbini, and T.T. Georgiou, Noninvasive estimation of tissue temperature via high-resolution spectral analysis techniques, IEEE Trans. on Biomedical Engineering vol. 52, pp. 221-228, 2005.

[37] R. T. Rockafellar, Convex Analysis, Princeton University Press, Princeton, NJ, 1970.

[38] W. Rudin, Principles of Mathematical Analysis 3rd ed., McGraw-Hill, New York, 1976.

[39] T. Söderström, P. Stoica, System Identification, Prentice Hall, New York, 1989.

[40] P. Stoica, R. Moses, Introduction to Spectral Analysis, Prentice Hall, New York, 1997.

[41] A. A. Stoorvogel, and J. H. van Schuppen, System identification with information theoretic criteria. In: Bittanti S, Picci G (eds) Identification, Adaptation, Learning: The Science of learning Models from Data. Springer, Berlin Heidelberg, 1996.

[42] P. Van Overschee, B. DeMoor, N4SID: Subspace algorithms for the identification of combined deterministic-stochastic systems, Automatica vol. 30, pp. 75-93, 1994.

[43] V. Vedral, The role of relative entropy in quantum information theory, Rev. Mod. Phys vol. 74, pp. 197-213, 2002.

[44] J. von Neumann, Mathematical Foundations of Quantum Mechanics, Princeton University Press, Princeton, 1955. 